\title{
Microenvironmental control of breast cancer subtype elicited by paracrine platelet derived growth factor-CC signaling
}

\author{
Pernilla Roswall ${ }^{\# 1}$, Matteo Bocci ${ }^{\# 1}$, Michael Bartoschek $\# 1$, Hong Li ${ }^{\# 2}$, Glen Kristiansen ${ }^{3}$, \\ Sara Jansson ${ }^{4}$, Sophie Lehn ${ }^{1}$, Jonas Sjölund ${ }^{1}$, Steven Reid ${ }^{1}$, Christer Larsson ${ }^{1}$, Pontus \\ Eriksson $^{4}$, Charlotte Anderberg ${ }^{1}$, Eliane Cortez ${ }^{1}$, Lao H Saal ${ }^{4}$, Christina Orsmark-Pietras ${ }^{5}$, \\ Eugenia Cordero', B Kristian Haller², Jari Häkkinen ${ }^{4}$, Ingrid JG Burvenich ${ }^{6}$, Elgene Lim ${ }^{7}$, \\ Akira Orimo ${ }^{8}$, Mattias Höglund ${ }^{4}$, Lisa Rydén ${ }^{4}$, Holger Moch ${ }^{9}$, Andrew M Scott ${ }^{6}$, Ulf \\ Eriksson $^{2}$, and Kristian Pietras ${ }^{1}$
}

\begin{abstract}
${ }^{1}$ Division of Translational Cancer Research, Department of Laboratory Medicine, Lund University, Lund, Sweden ${ }^{2}$ Division of Vascular Biology, Department of Medical Biochemistry and Biophysics, Karolinska Institutet, Stockholm, Sweden ${ }^{3}$ Institute of Pathology, University Hospital Bonn, Bonn, Germany ${ }^{4}$ Division of Oncology and Pathology, Department of Clinical Sciences, Lund University, Lund, Sweden ${ }^{5}$ Division of Clinical Genetics, Department of Laboratory Medicine, Lund University, Lund, Sweden ${ }^{6}$ Olivia Newton-John Cancer Research Institute, and School of Cancer Medicine, La Trobe University, Melbourne, Australia ${ }^{7}$ Garvan Institute of Medical Research, and University of NSW, Sydney, Australia ${ }^{8}$ Department of Pathology and Oncology, Juntendo University School of Medicine, Tokyo, Japan ${ }^{9}$ Department of Pathology and Molecular Pathology, University Hospital Zürich, Zürich, Switzerland

\# These authors contributed equally to this work.
\end{abstract}

\section{Abstract}

Breast tumors of the basal-like, hormone receptor-negative, subtype remain an unmet clinical challenge, as patients exhibit a high rate of recurrence and poor survival. Co-evolution of the malignant mammary epithelium and its underlying stroma instigates cancer-associated fibroblasts (CAFs) to endorse most, if not all, hallmarks of cancer progression. Here, we delineate a

Users may view, print, copy, and download text and data-mine the content in such documents, for the purposes of academic research, subject always to the full Conditions of use:http://www.nature.com/authors/editorial_policies/license.html\#terms

Correspondence should be addressed to Kristian.Pietras@med.lu.se.

Author contributions

PR generated and analyzed data, and conceived the study, M.Bocci generated and analyzed data, M.Bartoschek generated and analyzed data, HL generated and analyzed data, GK generated and analyzed data, SJ generated and analyzed data, SL generated and analyzed data, JS generated and analyzed data, CL generated and analyzed data, PE generated and analyzed data, SR generated and analyzed data, CA generated and analyzed data, E.Cortez generated and analyzed data, LHS generated and analyzed data, COP generated and analyzed data, E.Cordero generated data, BKH generated and analyzed data, JH generated and analyzed data, IJGB generated data, EL generated data, AO provided exclusive reagents, MH analyzed data, LR analyzed data and provided exclusive reagents, HM analyzed data and provided exclusive reagents, AMS analyzed data and provided exclusive reagents, UE analyzed data, provided exclusive reagents and conceived the study, KP generated and analyzed data, conceived the study, managed the study, and wrote the manuscript.

Competing Financial Interest

KP, UE and PR are named inventors on PCT application\# PCT/EP2016/077295 relating to the findings of the current study. KP, UE and AMS are shareholders of Paracrine Therapeutics that develop inhibitory agents to PDGF-CC. 
previously unappreciated role for CAFs as determinants of the molecular subtype of breast cancer. We identified a paracrine cross-talk between cancer cells expressing platelet-derived growth factor (PDGF)-CC and CAFs expressing the cognate receptors in human basal-like mammary carcinomas. Genetic or pharmacological intervention with PDGF-CC activity in mouse models of cancer resulted in conversion of basal-like breast cancers into a hormone receptor-positive state that conferred sensitivity to endocrine therapy in previously impervious tumors. We conclude that specification of the basal-like subtype of breast cancer is under microenvironmental control and therapeutically actionable in order to achieve sensitivity to endocrine therapy.

Breast cancer is a heterogeneous disease manifested as a range of different molecular subtypes of clinical relevance identified by gene expression analyses or biomarker expression 1,2. Breast tumors of the luminal subtypes (A or B) are the most common and characterized by expression of hormone receptors for estrogen (ERa) and/or progesterone (PgR), whereas $10-15 \%$ of women carry tumors of the basal-like subtype that do not express hormone receptors 3 . The prognosis and predictive characteristics of each breast cancer subtype are reflected by the adjuvant therapy options that are offered for that particular group of patients 4-6. Patients carrying luminal tumors typically benefit from treatment with drugs impinging on ERa signaling, such as tamoxifen, fulvestrant or aromatase inhibitors 7. In contrast, out of all breast carcinomas, the basal-like tumors have the highest recurrence rate, the shortest time to recurrence and worst overall survival rate due to a paucity of therapeutic targets $1,3,8,9$. Thus, novel treatment approaches for basal-like breast cancer patients are urgently required.

The breast cancer subtypes are defined by characteristics of the malignant cells. However, this tumor cell-centric view does not take into account the context in which cancer cells subsist. As the cancerous lesion progresses, the surrounding microenvironment co-evolves into an activated state through continuous paracrine communication, thus creating a dynamic signaling circuitry that promotes malignant initiation and progression 10 . Hence, from a therapeutic perspective, tumors must be regarded as multi-cellular organs. Cancer-associated fibroblasts (CAFs) are a major constituent cell type of the tumor stroma and are known to support many different aspects of tumor growth and progression by secretion of growth stimulatory, angiogenic, immune-modulatory, and pro-invasive factors 11,12 . However, the potential of CAFs as therapeutic targets or prognostic/predictive biomarkers is still debated, illustrating the possibility that CAFs represent a heterogenous group of cells with diverse and opposing functions 13,14. Indeed, a recent study defined histological subtypes also of the fibroblastic stroma of breast carcinomas, based on morphological criteria and marker expression 15 .

We have previously demonstrated a role for signaling by platelet-derived growth factors (PDGFs) in the recruitment and functionality of CAFs in solid tumors, such as melanoma and cervical carcinoma 16,17. While CAFs have been reported to express both PDGF receptors, i.e. PDGFRa and PDGFR $\beta$, specificity arises from the preference of receptor activation by the five different PDGF ligand isoforms (PDGF-AA, -AB, -BB, -CC, -DD). The in vivo specificity of one of the novel PDGF isoforms, PDGF-CC, remains undetermined, although in vitro studies suggest that PDGF-CC may activate PDGFRa 
homodimers, as well as PDGFRa $/ \beta$ heterodimers 18,19 . Herein, we delineate a previously unappreciated role for paracrine signaling by PDGF-CC in CAFs as a determinant of breast cancer prognosis and treatment response through specification of the molecular subtype of the tumor. In translational efforts, we found that PDGF-CC expression in the malignant epithelium of breast cancer was associated with a hormone receptor-negative state; this finding was replicated in independent cohorts of patients. Genetic or pharmacological targeting of PDGF-CC in experimental mouse models resulted in conversion of basal-like breast tumors into an ERa-positive state that conferred sensitivity to endocrine therapy in previously impervious tumors. Our work sheds light on previously unknown mechanisms that define breast cancer subtype and has significant therapeutic implications for patients with basal-like breast tumors.

\section{Results}

\section{PDGF-CC is an independent prognostic factor for poor survival in breast cancer}

To investigate the expression pattern of PDGF-CC in human breast cancer, we performed immunostaining of a tissue microarray containing 890 tumor specimens, as well as normal breast tissues (The Zürich cohort 20; for patient characteristics, see Supplementary Table 1). The expression of PDGF-CC in normal breast tissue was limited to myoepithelial/basal cells and endothelial cells in capillaries, whereas PDGF-CC expression was not detected in luminal epithelial cells (Fig. 1a-b). In breast tumors, PDGF-CC was expressed by malignant cells, intra-tumoral capillaries and stromal fibroblasts (Fig. 1c). Notably, PDGF-CC immunoreactivity was most conspicuous at the border between stromal tissue and malignant epithelium (Fig. 1d). Next, we evaluated the staining intensity for PDGF-CC independently for the epithelial and stromal compartment (for scoring schemes, see Supplementary Fig. 1ab) and correlated these findings to clinico-pathological parameters. Both stromal and malignant epithelial expression of PDGF-CC were positively correlated to the grade and proliferative index of the tumor, but not to the stage or lymph node status (Supplementary Table 2). Stromal immunoreactivity for PDGF-CC was not associated with patient outcome in Kaplan-Meier analysis, or identified as a risk factor for poor survival in univariate Cox regression analysis (Supplementary Fig. 1c and Table 1). By contrast, moderate to high expression of PDGF-CC in malignant cells (score of $1+$ to $3+$ ) was found to be a statistically significant prognostic factor for poor survival (Fig. 1e and Table 1). Importantly, multivariable Cox regression analysis adjusting for established clinical risk factors, such as age at diagnosis, stage, grade, lymph node status, hormone receptor expression, among others, demonstrated that epithelial expression of PDGF-CC served as an independent prognostic factor for poor survival in breast cancer (Table 1).

Our findings were corroborated in a second breast cancer cohort of 550 patients (The Lund cohort 21,22; for patient characteristics see Supplementary Table 3), in which high epithelial expression of PDGF-CC was correlated to grade and associated with poor survival (Supplementary Table 4 and Supplementary Fig. 1d). Both receptors of the PDGF family, i.e. PDGFR $a$ and PDGFR $\beta$, were prominently and uniformly expressed by stromal fibroblasts in tumors from the Lund cohort (Supplementary Fig. 1e-f; PDGFRa and PDGFR $\beta$ expressed by CAFs in 482/489 (98.6\%) and 480/480 (100\%) of evaluable tumors, 
respectively). These findings indicate that malignant cells engage in a functional paracrine communication with mesenchymal cells of the tumor microenvironment through PDGF signaling.

\section{PDGF-CC is functionally important for the growth of experimental breast cancer in vivo}

To investigate the functional aspects of PDGF-CC expression in the context of mammary gland tumorigenesis, we generated a genetically engineered mouse model of breast cancer based on the widely studied and relevant MMTV-PyMT mouse 23,24 intercrossed with mice carrying an insertion of a lac Z cassette that inactivates the gene for PDGF-CC 25 ( $P d g f c^{-1-}$ mice). Visualization of PDGF-CC, PDGFRa and PDGFR $\beta$ expression in tumors of MMTVPyMT mice demonstrated faithful recapitulation of the expression pattern in human breast cancers, thus establishing the existence of a paracrine PDGF circuitry between malignant cells and stromal fibroblasts (Supplementary Fig. 2a-d). Strikingly, genetic deficiency for Pdgfc severely impacted on the growth of mammary tumors in MMTV-PyMT mice (Fig. 1f). Control mice presented with tumors of an average size of $220 \pm 47 \mathrm{~mm}^{3}$, whereas deficiency for a single or both copies of $P d g f c$ significantly reduced the average tumor size to $96 \pm 41$ $\mathrm{mm}^{3}$ and $87 \pm 71 \mathrm{~mm}^{3}$, respectively (Fig. 1f). Genetic deficiency for $P d g f c$ was also associated with a significantly longer tumor latency, as well as prolonged survival of the mice (Supplementary Fig. 3a-b). In line with the findings in human breast cancer, histological analyses revealed that tumors from $P d g f^{-/-}$mice were of lower grade compared to tumors from age-matched control animals (Fig. 1g-i) and had significantly increased areas of necrosis (Fig. 1g and j-k). We also observed a trend towards fewer pulmonary metastases in 14 weeks old tumor-bearing mice in the absence of PDGF-CC signaling (Supplementary Fig. 3c). However, this was most likely due to the delayed onset of disease, as a cohort of wt mice with grade-matched tumors to the Pdgfc-deficient cohort displayed a similar metastatic burden as the mice lacking Pdgfc (Supplementary Fig. 3c).

To ascertain that the delayed tumor development in $P d g f c$-deficient mice was not due to developmental defects, we transplanted fragments of tumors from MMTV-PyMT; Pdgfc $f^{+/+}$ and MMTV-PyMT; Pdgfc ${ }^{-/}$mice orthotopically into the mammary fat pad of wt mice. Whereas all (21/21) transplanted wt tumor fragments established as growing tumors, only about half $\left(11 / 21, \chi^{2}\right.$ test $\left.\mathrm{p}=0.003\right)$ of the transplanted $P d g f c$-deficient fragments gave rise to tumors. Consistent with our findings in the transgenic setting, transplanted $P d g f c$-deficient tumors displayed a dramatically hampered growth, compared to Pdgfc-proficient tumors (Fig. 11). In addition, whereas two independently isolated cell lines from tumors in wt MMTV-PyMT mice (PeRo-Bas1 and PeRo-Bas2 cells) readily gave rise to exponentially growing tumors following orthotopic transplantation into the mammary fat pad of wt mice, cell lines from tumors in MMTV-PyMT; Pdgfc ${ }^{-/-}$mice (PeRo-Lum1 cells and PeRo-Lum2 cells) failed to efficiently establish as palpable tumors (Supplementary Fig. 3d-e).

\section{Pdgfc deficiency results in a blunted fibrotic and angiogenic response in the tumor microenvironment}

Histological analyses revealed considerable differences in the tumor architecture between MMTV-PyMT; $P d g f c^{+/+}$and MMTV-PyMT; $P d g f c^{-/}$mice. Masson's tri-chrome staining of tumor sections demonstrated a severely reduced deposition of intra-tumoral collagen in the 
matrix of $P d g f c$-deficient tumors, consistent with the notion that PDGF-CC signaling promotes the recruitment and/or activation of stromal fibroblasts in the breast tumor microenvironment (Fig. 1m-n). In support of this notion, quantitative RT-PCR analysis showed reduced abundance of mRNA transcripts of prototypical markers for CAFs, such as a-smooth muscle actin (Acta2), fibroblast-specific protein 1 (S100a4) and PDGFRa (Pdgfra) in Pdgfc ${ }^{-/}$tumor samples (Fig. 1o, Student's t-test $\mathrm{p}<0.01$ for each marker). Tumors from MMTV-PyMT; $P d g f \mathcal{C}^{-/-}$mice exhibited significantly increased levels of hypoxia, as evidenced by immunostaining for HIF-1a, the master transcriptional regulator of hypoxia (Supplementary Fig. 4a-c). Consistently, quantitative RT-PCR analysis revealed a $65 \%$ reduction in vascular endothelial growth factor-A expression levels in the absence of PDGF-CC (Supplementary Fig. 4d). Furthermore, in line with the increased abundance of necrotic area, these tumors were severely hemorrhagic (Supplementary Fig. 4e). Neutralization of PDGF-CC by administration of the inhibitory antibody $6 \mathrm{~B} 3$ to tumorbearing SCID mice delayed the growth and angiogenic response of orthotopic basal-like MDA-MB-231 tumors (Supplementary Fig. 5a-c). Taken together, genetic and pharmacological blockade of PDGF-CC corroborate a role for paracrine signaling in breast tumor growth, CAF recruitment and angiogenesis.

\section{Expression of PDGF-CC in breast tumors is associated with a basal-like molecular subtype}

To elucidate the molecular signature of $P d g f c$-deficiency, we performed transcriptional analysis of tumors derived from MMTV-PyMT; $P d g f c^{+/+}$and MMTV-PyMT; Pdgfc $c^{-/}$mice using a quantitative RT-PCR array designed to analyze a targeted panel of genes instrumental in breast tumor development and progression. The most differentially regulated gene was found to be Foxa1, which was expressed on average 17.2-fold higher in whole tumor lysates from $P d g f c$-deficient MMTV-PyMT mice compared to wt mice, and 6.5-fold higher in cell lines isolated from tumors lacking Pdgfc (Fig. 2a and Supplementary Fig. 6a). The association between FoxA1 expression and loss of PDGF-CC was further corroborated at the protein level in whole tumor lysates of mammary carcinomas from MMTV-PyMT; Pdgfc $^{+/-}$mice and MMTV-PyMT; Pdgfc $\mathcal{C}^{-/-}$mice (Supplementary Fig. 6b).

Next, we set out to determine the functional implications of high expression of Foxal in $P d g f c$-deficient breast tumors. Analysis of transcriptional profiles of 1086 breast tumors collected within The Cancer Genome Atlas network 26 revealed that expression of FOXA1 was highly correlated with a non-basal-like molecular subtype (Supplementary Fig. 6c), confirming previous studies 1,27,28. In accordance with the association between FOXA1 and luminal subtype breast cancer, tumors from $P d g f c$-deficient MMTV-PyMT mice with high levels of FoxA1 protein also exhibited robust expression of ERa, as judged by western blot analysis (Supplementary Fig. 6b). Given the fact that Foxa1 was found to be upregulated in experimental breast tumors in the absence of $P d g f c$, we investigated the correlation between $F O X A 1$ and $P D G F C$ in human breast cancer. Mining of transcriptional data from a panel of 51 breast tumor cell lines 29 demonstrated expression of FOXA1 as a specific feature of cell lines of the luminal subtype, whereas basal-like cell lines did not express meaningful levels of FOXA1, as expected (Fig. 2b). In contrast, high expression of $P D G F C$ was predominantly observed in breast tumor cell lines of the basal-like subtype, but not in cells of luminal subtype origin (Fig. 2c). Indeed, the expression of PDGFC and 
FOXA1 exhibited a statistically significant inverse correlation in this panel of cell lines (Fig. $2 \mathrm{~d}$ ). The enrichment for expression in basal-like breast tumor cell lines was exclusively observed for $P D G F C$, and not for other members of the PDGF family (Fig. 2e). To investigate whether the absence of $P D G F C$ was associated with a full luminal gene regulatory program, we analyzed the enrichment of binding sites for the luminal-defining transcription factors ESR 1, FOXA1, and GATA3, as derived from public datasets of ChIPseq analysis of luminal MCF7 and T47D cells 30, in the most differentially expressed genes in $\mathrm{E}_{2}$-stimulated $P d g f c$-deficient PeRo-Lum1 cells compared to Pdgfc-proficient PeRo-Bas1 cells. While we were unable to demonstrate further induction of Esr target genes by $\mathrm{E}_{2}$ in PeRo-Lum1 cells, the basal expression level was superior in PeRo-Lum1 cells compared to PeRo-Bas1 cells, suggesting a constitutive activation of ERa signaling. In relation to $10^{5}$ randomly permuted gene lists of 1000 genes, both the top 1000 overexpressed genes, and the top 1000 underexpressed genes, in the $P d g f c$-deficient cells were significantly enriched for orthologous human genes with ESR1, FOXA1, and GATA3 binding sites in both MCF7 and T47D cells (Fig. 2f-k and Supplementary Fig. 6d-g), indicating activation of a global luminal gene regulatory program in the absence of $P d g f c$. In further support of the existence of an activated ERa pathway in PeRo-Lum1 cells, Gene Set Enrichment Analysis (GSEA) demonstrated that treatment with tamoxifen resulted in enrichment of documented ERa target genes or genes with documented sensitivity to tamoxifen (Supplementary Fig. 7). Additionally, tamoxifen treatment of $P d g f \mathcal{c}$-deficient breast cancer cells induced a gene profile related to decreased cell cycle progression, demonstrating manifestation of functional ERa signaling in PeRo-Lum1 cells (Supplementary Fig. 7). Finally, we also submitted a list of genes ranked by their correlation to the expression of $P D G F C$ in breast tumors included in The Cancer Genome Atlas to GSEA. Consistently, genes that were inversely related to $P D G F C$ expression were part of datasets identifying ERa target genes, whereas genes that were positively correlated to $P D G F C$ expression were part of datasets related to inhibition of ERa signaling (Supplementary Fig. 8). Jointly, our analyses utilizing a diverse set of approaches strongly point to an association between the absence of expression of PDGFC and a luminal phenotype characterized by functional ERa signaling.

Next, we further investigated the association of PDGF-CC expression with the molecular subtype of breast cancer by analysis of expression of basal-like and luminal markers in the Zürich cohort of human breast tumors by immunostaining (Table 2 and Supplementary Fig. 9). Strikingly, PDGF-CC showed a highly significant correlation to the basal-like markers cytokeratin (CK) 5/6 and epidermal growth factor receptor (EGFR), while low expression of PDGF-CC denoted hormone receptor-positive tumors expressing FOXA1, ERa and PgR (Table 2). The inverse correlation between PDGF-CC and hormone receptors was further corroborated in the Lund cohort, in which the relationship was also established in synchronous lymph node metastases and asynchronous distant metastases (Fig. 21-m and Supplementary Table 5). In primary tumor epithelium, only $7.8 \%$ of the luminal A subgroup were positive for PDGF-CC, whereas $71.9 \%$ of triple negative tumors were positive (Supplementary Table 6). Taken together, we have revealed a close association between expression of PDGF-CC and hormone receptor-negative breast tumors in cohorts of human patients. 


\section{A paracrine signaling circuit in stromal fibroblasts established by PDGF-CC determines the molecular subtype of breast tumor cells}

We then investigated the molecular mechanisms through which paracrine signaling by epithelium-derived PDGF-CC was associated with basal-like features of breast tumors. Conditioned medium from the immortalized breast CAF cell line CAF2 31 significantly reduced the sensitivity of PeRo-Lum1 mammary carcinoma cells with luminal features isolated from a tumor in a MMTV-PyMT; $P d g f c^{-/-}$mouse to tamoxifen-induced growth arrest (Fig. 3a). This finding suggested that secreted factors from stromal fibroblasts conferred resistance to endocrine therapy. Next, we stimulated CAF2 cells with PDGF-CC and performed global transcriptional analysis. A list of statistically significantly upregulated genes (fold change >1.4) was generated and filtered for Gene Ontology terms "Extracellular space" and "Extracellular region". The resulting list of genes was used to generate hypotheses concerning which factors mediated the effect of PDGF-CC, and candidate secreted proteins that were highly induced by PDGF-CC were tested for their ability to regulate the sensitivity of luminal breast cancer cells to the action of tamoxifen. The most significant findings, stanniocalcin 1 (Stcl), hepatocyte growth factor $(\mathrm{Hgf})$ and insulin growth factor binding protein 3 ( $I g f b p 3$ ) were validated by quantitative RT-PCR to ensure robust induction by PDGF-CC in CAF2 cells after 48h of stimulation (Supplementary Fig. 10). Strikingly, pre-treatment with a cocktail of the CAF-derived factors HGF, IGFBP3 and STC1 reduced the sensitivity of PeRo-Lum1 cells to tamoxifen-induced growth arrest (Fig. 3b). We also assessed whether stimulation of PeRo-Lum1 cells with HGF, IGFBP3 and STC1 converted their ERa-positive phenotype into ERa-negative. Indeed, while each factor alone affected the expression of luminal markers Esr1, Foxal and Gata3 to some degree, the concerted action of HGF, IGFBP3 and STC1 substantially suppressed the luminal-like features of $P d g f c$-deficient mammary carcinoma cells (Fig. 3c-e). The expression of HGF, IGFBP3 and STC1 by CAFs populating the stroma of tumors from MMTV-PyMT; Pdgfc ${ }^{+/+}$ mice was further confirmed by immunostaining (Fig. 3f-h). Reassuringly, in breast tumors from the TCGA cohort, gene expression of $P D G F C, H G F$ and $I G F B P 3$ were all significantly, albeit modestly, correlated to each other and to $F O X C 1$, a transcriptional regulator of the basal-like phenotype 32 (Fig. 3i), suggesting commonality in their regulation also in human tumors. By contrast, and in line with our observations, the expression of $P D G F C, H G F$ and IGFBP3 were inversely correlated to the luminal markers FOXA1, ESR 1 and $G A T A 3$ (Fig. 3i). In support, a cohort of 43 triple-negative breast cancer specimens displayed a close correlation between the expression of HGF, IGFBP3 and STC1 in the tumor stroma (Supplementary figure 11a-c and Supplementary table 7). All three factors were also variably expressed by the tumor epithelium, but a lack of close correlation in expression indicates distinct mechanisms of gene regulation (Matteo Bocci, data not shown). Similarly, immunostaining and scoring of a representative selection of 58 human breast tumors included in the Tam2Y cohort33 for epithelial PDGF-CC, stromal HGF, stromal IGFBP3 and stromal STC1 demonstrated their close correlation with each other and with a basal-like, ERa-negative phenotype (Supplementary table 8). In summary, paracrine signaling by PDGF-CC stimulates the expression of HGF, IGFBP3 and STC1 in breast CAFs from luminal tumors, leading to a malignant cell phenotype denoted by lack of ERa and other luminal markers, and a reduced sensitivity to tamoxifen. 


\section{Genetic or pharmacological targeting of PDGF-CC sensitizes ERa-negative breast tumors to hormone therapy}

ERa expression, which confers sensitivity to endocrine therapy such as tamoxifen, is the most important distinguishing clinical feature of luminal subtype breast tumors. We therefore investigated whether targeting of PDGF-CC would convey sensitivity to endocrine therapy to previously impervious ERa-negative breast tumors in vivo. Wildtype FVB/N mice bearing orthotopically transplanted tumors from MMTV-PyMT; $P d g f c^{+/+}$or MMTVPyMT; $P d g f c^{-/-}$mice were treated daily with tamoxifen starting from the time at which tumors were readily palpable. As expected, due to the lack of ERa expression at this late stage of malignant development, the wt tumors of tamoxifen-treated MMTV-PyMT mice continued to grow at a similar rate as tumors from control-treated mice (Fig. 4a). In sharp contrast to the progressive growth of all wt tumors, $P d g f c$-deficient tumors were severely growth-retarded upon treatment with tamoxifen and 71\% (5/7) of the mice exhibited a complete or partial response (defined as full regression or shrinkage of tumor volume by more than 30\%, respectively), in agreement with their expression of ERa (Fig. 4b). At the end of the trial, tumors from tamoxifen-treated mice devoid of paracrine PDGF-CC signaling had a reduced volume by an average of $17 \%$, whereas control-treated mice presented with tumors that had grown by $624 \%$ since treatment initiation, evidently revealing functional sensitization of ERa-signaling within Pdgfc-deficient breast tumors (Fig. 4b).

To conclusively demonstrate the utility of agents targeting PDGF-CC to sensitize breast tumors of the basal-like subtype to the action of tamoxifen, we implanted the triple-negative patient-derived xenografts (PDX) 12.58 and 14.32, as well as the basal-like breast cancer cell line MDA-MB-231 orthotopically into immunocompromised mice. Indeed, analysis revealed that 12.58 and MDA-MB-231 tumors that originally did not express meaningful levels of ERa, substantially upregulated expression of ERa upon treatment with 6B3 as judged by immunostaining, corroborating the role of paracrine signaling by PDGF-CC in establishing ERa negativity in basal-like subtype breast tumors (Fig. 4c-f and Supplementary Fig 12a-d). The upregulation of ERa expression in 12.58 and MDA-MB-231 tumors following PDGF-CC blockade was not uniform, but rather occurred focally in differentiated nests of malignant cells (Supplementary Fig. 12a-d). Importantly, 6B3treatment was sufficient in itself to push $3 / 8$ (37.5\%) of the basal-like PDX tumors above the $1 \%$ threshold of ERa positivity used clinically to mandate treatment with endocrine therapy 34. As expected, treatment with tamoxifen together with a control antibody was unable to influence the growth of fully established 14.32 or MDA-MB-231 tumors (Fig. 4g-h). By contrast, and in good agreement with the converted ERa-positive phenotype upon neutralization of PDGF-CC, combined administration of tamoxifen and 6B3 led to significant growth retardation of both 14.32 and MDA-MB-231 tumors (Fig. 4i-j). The frequency of ERa-positive cancer cells diminished in MDA-MB-231 tumors following tamoxifen therapy in combination with the 6B3 antibody, demonstrating that the induction of ERa caused by neutralization of PDGF-CC indeed invoked sensitivity to the action of tamoxifen (Fig. 4f). Similarly, pharmacological blockade of PDGF-CC also brought about a growth delay of MDA-MB-231 xenografts in face of treatment with the aromatase inhibitor letrozole (Supplementary Fig. 12e-f), suggesting that the strategy is widely applicable to 
sensitize basal-like tumors to endocrine therapies such as tamoxifen and letrozole with diverse mechanisms of action. Conversely, mice xenografted with ERa-expressing luminal MCF7 cells with ectopic expression of Pdgfc did not respond to tamoxifen therapy, in contrast to mice implanted with the parental clone (Fig. 4k). Jointly, our studies thus establish functional sensitization of ERa-negative tumors to endocrine therapy as a consequence of conversion into an ERa-positive state through blockade of paracrine signaling by PDGF-CC.

\section{Discussion}

We have revealed a paracrine signaling network manifested in the basal-like breast tumor microenvironment, in which tumor epithelium-derived PDGF-CC orchestrates specification of an ERa-negative phenotype through activation of CAFs that are induced to secrete HGF, IGFBP3 and STC1 (Fig. 41). Blockade of PDGF-CC, either by genetic or pharmacological means, prompted sensitization of previously impervious breast tumors to the action of endocrine therapy by inducing the expression of ERa (Fig. 41). Our findings unexpectedly identify CAFs as functional regulators of the molecular subtype of breast cancer, and offer novel therapeutic opportunities for a patient group in need of improved treatment options.

The distinguishing features of luminal and basal-like breast cancer cells have been thought to signify different cells-of-origin in the normal mammary gland, i.e. luminal progenitor cells and breast epithelial stem cells, respectively 35 . However, recent studies describe a high degree of plasticity and heterogeneity in the definition of luminal or basal-like tumors. Interconversion of luminal or basal-like tumor cells has been demonstrated to occur efficiently in vitro, indicating a common progenitor cell origin 36. Indeed, based on gene expression analysis and functional studies, luminal progenitor cells have been proposed as a common source of luminal and basal-like breast carcinomas 37-40. Moreover, a phenotypic switch from basal-like to luminal breast cancer can be achieved by introduction of the genes for ERa, FOXA1 or GATA3, whereas a transition from a basal-like state into a luminal state can result from epigenetic reprogramming 41-43. Interestingly, cell cultures that were isolated from tumors in Pdgfc-deficient mice exhibited constitutive ERa signaling and a luminal phenotype, while retaining sensitivity to tamoxifen. Such tools may hold important clues as to the nature of the epigenetic programming of the molecular subtype of breast cancer. In line with our work, a recent study demonstrated development of tumors with differential ERa expression in lesions grafted from the same luminal PDX, but at different anatomical sites, i.e. the mammary fat pad or the milk ducts, suggesting profound microenvironmental regulation 44. Rather than searching for new drugs targeting (epi)genetic alterations in basal-like breast tumors, pharmacological strategies for the induction of a switch from hormone receptor-negative breast cancer into a luminal subtype would offer established and effective treatment options for a large patient group in need of improved therapy. As such, our studies demonstrating that luminal/basal-like specification is under control of the tumor microenvironment through regulation of FOXA1, ERa and GATA3, afford novel targeting opportunities by interfering with paracrine signaling between malignant cells and CAFs. 
Recent studies have suggested that PDGFRa expression may be a feature of malignant cells exhibiting stem cell properties and/or have undergone epithelial-to-mesenchymal transition in breast cancer 45,46 . However, we have previously shown that malignant cell expression of PDGFRa is not a universal feature of human mammary carcinomas in the Lund cohort, as expression of PDGFRa in overt cancer cells was only found in $20 \%$ of cases 47 . In contrast, virtually all breast tumors in the same cohort harbored CAFs with expression of PDGFRa and PDGFR $\beta$, strongly indicating that the paracrine mode of signaling by PDGF-CC, and not autocrine signaling, is the most prominent route of signal transduction in human breast cancers. Stimulation of breast CAFs with PDGF-CC induced the expression of HGF, IGFBP3 and STC1. Previous conditional PDGFRa gene targeting studies have demonstrated a reduced expression of HGF and collagen by fibroblasts, lending support to our findings 48 . Expression of the receptor for HGF, MET, has also been associated with basal-like breast cancer, in agreement with our study 49-52. Intriguingly, constitutive activation of MET signaling drives commitment of luminal progenitor cells to a basal-like cell fate 53. In the case of IGFBP3, the association with hormone receptor-negative breast cancers and poor survival is already well established, although a causal relationship has not been reported previously 54 .

Mesenchymal cells are highly plastic entities. Normal fibroblasts are described to counteract tumorigenesis by repressing cancer initiation 14,55,56. However, co-evolution of the malignant epithelium and its underlying stroma instigates activation of CAFs into a phenotype endorsing most, if not all, hallmarks of cancer progression 11,57. Abundant evidence thus supports the utility of CAFs as drug targets by virtue of their tumor-promoting abilities. Indeed, a recent study identified a subset of CD146-negative CAFs that confer resistance to endocrine therapy in luminal breast cancer58. However, investigations of pancreatic adenocarcinoma, a cancer type harboring severe desmoplasia, caution against indiscriminate blockade of CAF recruitment and/or activation 59,60. By the use of inhibitors of hedgehog signaling, or by eradication of a-SMA-expressing CAFs, genetically engineered pancreatic adenocarcinomas were allowed to thrive in an unrestricted manner. Given the diverse functions described, it is thus likely that CAFs represent a heterogenous population harboring both growth-promoting and -inhibitory subsets. Nevertheless, despite sometimes aggravating tumorigenesis, blockade of CAF recruitment sensitized pancreatic tumors to anti-angiogenic therapy or immune checkpoint inhibitors 59,60. Similarly, we found that blockade of signaling by PDGF-CC held utility as a combination partner to endocrine therapy in experimental mammary carcinomas. Therapeutic targeting of CAFs may thus best be exploited in joint treatment regimens with drugs impinging on multiple cell types and signaling pathways that collectively sustain malignant growth.

The work presented here identifies a hitherto unappreciated role of the tumor microenvironment in the specification of the molecular subtype of breast cancer and distinguishes PDGFRa-expressing CAFs as a promising pharmacological target to achieve manifestation of ERa in mammary carcinomas. As such, our discovery of neutralization of PDGF-CC as an efficacious combination partner to endocrine therapy justifies clinical evaluation for the treatment of patients suffering from basal-like breast cancer. 


\section{Online Methods}

Further information on experimental design and reagents can be found in the accompanying Life Sciences Reporting Summary

\section{Patient cohorts}

The Zürich cohort includes tissues from 890 patients with primary invasive breast cancer, diagnosed at the Institute of Surgical Pathology, UniversitätsSpital Zürich, between 1965 and 2004 (median July 1999). All patients enrolled voluntarily under Institutional Review Board-approved protocols and sample donors gave written informed consent. The ethics committee SPUK surgical-anesthetic-pathology at university hospital of Zürich, Switzerland, approved this study with reference number: StV 12-2005. For all these patients follow-up data from the cantonal cancer registry were available; patients without follow-up information were not considered 61. Additionally, 69 normal tissues were analyzed. The Lund cohort includes patients with primary breast cancer from a prospective, observational trial evaluating various prognostic markers (local ethical review board reference numbers LU692-99 and LU247-01); clinical-pathological and follow-up data have previously been reported 62,63. Informed consent was obtained from all patients. Breast cancer-specific mortality was used as the endpoint and data on survival was retrieved from the Swedish Register of Causes of Death (Central Statistics Office). All events until December 2010 were recorded. The specimens from the Tam2Y cohort consists mainly of postmenopausal patients with stage II breast carcinoma from the South Swedish Health Care Region between 1985 and 199464. For the current study, a representative subset of 58 tumors from the cohort was analyzed. The cohort of human triple-negative breast cancers was obtained commercially (Abcam, ab214756) and consisted of 43 evaluable specimens.

\section{Immunohistochemistry on human tumor tissue arrays}

To assess PDGF-CC expression in clinical samples, the monoclonal PDGF-CC antibody 6B3 $(2 \mu \mathrm{g} / \mathrm{ml}$ ) was used on an automated Ventana platform (protocol CC1m for pretreatment, UView HRP detection system). Specific immuno-reactivity was fully blocked by an excess of active PDGF-CC. The basal cell marker cytokeratin CK5/6 (1:25, clone cocktail D5/16B4, Dako), HER2 (1:50, clone 10A7 Novocastra, UK), EGFR (pre-diluted, clone 3C6 Ventana) and Ki-67 (1:20, clone Mib-1 Dako) were processed in parallel. Additionally, immunostaining was performed for HGF (1:100, Abcam ab83760), IGFBP3 (1:100, Santa Cruz Biotechnology sc-9028), and STC1 (1:100, Santa Cruz Biotechnology sc-30183).

\section{Animals}

All animal experiments were approved by the Ethical Committee for Animal Experimentation (Stockholm Norra djurförsöksetiska nämnd, application N96/11, and Lund/ Malmö djurförsöksetiska nämnd, application M142/13) or by the Austin hospital Animal Ethics Committee. Experimentation were conducted in compliance with the Australian Code for the care and use of animals for scientific purposes. FVB/N-Tg(MMTV-PyVT) ${ }^{634 M u l / J}$ transgenic mice have been described previously 65 and were purchased from The Jackson Laboratory. The presence of the MMTV-PyMT transgene and the generation of heterozygous and homozygous knock-out Pdgfc 66 offspring were verified by genotyping. 
DNA was prepared from either ear or tail biopsies according to a common tissue lysis, nucleic acid extraction and purification protocol.

MMTV-PyMT primer pair (5' to 3'): GGAAGCAAGTACTTC ACAAGGG and GGAAAGTCACTAGGAGCAGGG.

\section{Pdgfc wild-type pair (5' to 3'): AGCTGACAT TTGATGAGAGAT and AGTAGGTGAAATAAGAGGTGAACA.}

\section{Pdgfc mutant pair (5' to 3'): CTC ATGTTCTCGTGACTCTGA and TAGCTAGTCGATACCGTCGA.}

Mice deficient for $P d g f \mathcal{C}$ were originally in the C57B1/6 background and backcrossed to the $\mathrm{FVB} / \mathrm{N}$ background for 10 generations before analysis was commenced. Female mice were used throughout the studies. In all cases, wt littermate mice were used as controls to genetically engineered mice.

\section{Animal experiments}

For transgenic MMTV-PyMT mice, tumor size of each of the 10 mammary glands were measured at 12 weeks of age using a caliper. Tumor volume was calculated as length $\times$ width $^{2} \times \pi / 6$. The endpoint for survival analysis was taken as the time at which the combined tumor burden surpassed the ethical guidelines in our permit.

For transplantation experiments, 3 weeks old FVB/N female mice were anesthetized and maintained under Isoflurane during the surgical procedure. A $4 \mathrm{~mm}$ incision under the nipple of the right abdominal mammary gland created a pocket where a $2 \times 2 \mathrm{~mm}$ tumor piece (pieces kept on ice derived from either MMTV-PyMT; $P d g f_{c} c^{+/+}$mice or MMTV-PyMT; $P d g f c^{-/-}$mice) was inserted. Suturing was performed with 6-0 Ethilon polyamide filament (Ethicon). Pain-killer and anti-inflammatory Rimadyl $(5 \mathrm{mg} / \mathrm{kg}$ body weight; Orion Pharma Animal Health) was injected i.p. at the end of the surgical procedure and for the following two days. Tumor growth was monitored and measured in sedated mice twice per week with a caliper.

Mouse mammary cell lines derived from wt or Pdgfc-deficient MMTV-PyMT mice established in our laboratory were orthotopically injected into the $4^{\text {th }}$ inguinal mammary gland of wt FVB/N mice. Tumor growth was monitored and measured in sedated mice twice per week with a caliper.

For therapeutic studies, $2 \times 10^{6}$ Human MDA-MB-231 cells were inoculated orthotopically in SCID mice. Mice had been randomly assigned before tumor inoculation to receive treatment with anti-PDGF-C (mouse monoclonal antibody clone 6B3) antibody or IgG2a isotype control antibody (Bio X Cell), which were delivered via i.p. injection twice a week (300 $\mu \mathrm{g}$ per week) starting from the day of tumor establishment. For therapeutic trials involving endocrine therapy, when a tumor was palpable (longest diameter $>3 \mathrm{~mm}$ ), mice were alternately assigned into the treatment groups in which mice were treated with tamoxifen (1 mg/dose via oral gavage daily, Sigma), dissolved in a vehicle of ethanol and 
corn oil (Sigma) by heating to $55^{\circ} \mathrm{C}$, or with vehicle alone. All therapeutic administrations were open-label.

For all transplantation experiments, tumor growth was monitored and measured in sedated mice twice a week with a caliper.

\section{Patient-derived xenografts}

A total of 8 mice (Nod Scid gamma, NSG) were implanted with 1-2 $\mathrm{mm}^{3}$ tumor pieces of patient-derived triple negative breast cancers with documented high expression of PDGF-CC (12.58, BRCA2 mutation, derived from liver metastasis, treatment resistant; 14.32, derived from primary tumor, treatment naïve). Two tumor pieces were implanted per mouse, in two mammary fat pads, one on each side of the mouse. Ten days after implantation, mice received anti-PDGF-CC therapy $(50 \mathrm{mg} / \mathrm{kg}$ ch6B3, three times per week) or vehicle control (PBS). Tumors were harvested and fixed in $10 \%$ formalin. Four $\mu \mathrm{m}$ sections of the paraffinembedded tumors were mounted onto SuperFrost ${ }^{\circledR}$ Plus slides (Menzel-Glaser, Braunschweig, Germany), deparaffinized and rehydrated prior to antigen retrieval for $36 \mathrm{~min}$ at $95^{\circ} \mathrm{C}$ in $\mathrm{CC} 1$ buffer (Ventana Medical Systems Inc, Tuscon, AZ, USA). Ready-to-use CONFIRM anti-estrogen receptor (ER) antibody, clone SP1 (Ventana Medical Systems Inc.) was applied to sections for $40 \mathrm{~min}$ at $36^{\circ} \mathrm{C}$. To allow visualization of the immunostaining, sections were incubated with the UltraView Universal DAB Detection Kit (Ventana Medical Systems Inc.) and counterstained with Mayer's haematoxylin. The staining was performed on board the Ventana BenchMark Ultra automated immunohistochemical staining instrument (Ventana Medical Systems Inc).

\section{Mouse tissue preparation, histology and immunostaining}

To preserve tissues for downstream analysis, mice were heart-perfused with PBS followed by $4 \%$ paraformaldehyde. For paraffin-embedding, organs were post-fixed in $4 \%$ paraformaldehyde for $12 \mathrm{~h}$ in $4{ }^{\circ} \mathrm{C}$. Paraffin-embedded sections were deparaffinized and rehydrated followed by antigen retrieval in citrate buffer (pH 6; DAKO) in a pressure cooker (ERa) or in $95^{\circ} \mathrm{C}$ water-bath for 20 minutes (STC1, IGFBP3 and HGF). Endogenous peroxidase activity was quenched with $3 \% \mathrm{H}_{2} \mathrm{O}_{2}$ in methanol for 10 minutes at room temperature, followed by washes with $0.1 \%$ BSA in PBS.

ERa staining required subsequent steps in M.O.M. blocking (Mouse on Mouse basic kit, Vectorlabs), CAS-block (Life Technologies) and M.O.M. diluent. The primary antibody against estrogen receptor ERa (1:200, clone 1D5; DAKO) was incubated in M.O.M. diluent. CAS-block was used for the blocking and incubation of primary antibodies against STC1 (1:200, sc-30183, Santa Cruz Biotechnology), IGFBP3 (1:200, sc-9028 Santa Cruz Biotechnology) and HGF (1:200, ab83760 Abcam). Primary incubation was performed overnight at $4{ }^{\circ} \mathrm{C}$ in a humidified chamber. After washing, appropriate secondary biotinylated antibodies and the $\mathrm{ABC}$ peroxidase system were used (ABC Elite standard kit, Vector Laboratories) with DAB as substrate (Vector Laboratories).

For cryopreservation, primary tumor and lungs were kept in $30 \%$ sucrose at $4{ }^{\circ} \mathrm{C}$ overnight, followed by embedding in Optimal Cutting Temperature medium (HistoLab). Frozen sections were fixed in ice-cold acetone, followed by blocking using Serum Free Protein 
Block (DAKO) for $>90$ minutes at room temperature. Primary antibodies directed against PDGFRa (PE-conjugated, 1:200, 12-1401 eBioscience) and PDGFR $\beta$ (1:200, 3169S, Cell Signaling) were incubated overnight at $4{ }^{\circ} \mathrm{C}$ in a humidified chamber. Appropriate Alexa488- fluorochrome-conjugated secondary antibody (Life Technologies) was used and sections were finally mounted using 4',6-diamidino-2-phenylindole (DAPI)-containing mounting media (Vector Laboratories).

Imaging was performed using an upright microscope (Eclipse E800; Nikon) equipped with Plan Fluor objectives $(10 \times, 0.30 \mathrm{NA} ; 20 \times, 0.50 \mathrm{NA} ; 40 \times, 0.75 \mathrm{NA}$; Nikon) at room temperature in air. Images were acquired using a SPOT RTKE camera using the SPOT advanced software (Diagnostic Instruments, Inc.).

\section{$\beta$-galactosidase staining}

Fresh frozen sections were left to dry at RT for 15 minutes before washing in permeabilization solution $\left(4 \mathrm{mM} \mathrm{MgCl}_{2}, 0.04 \% \mathrm{NP}-40,0.02 \%\right.$ deoxycholic acid sodium salt in PBS) $2 \times 30$ minutes. Sections were further subjected to X-gal solution $(5 \mathrm{mM} \mathrm{K} 3 \mathrm{Fe}(\mathrm{CN}) 6$, $5 \mathrm{mM} \mathrm{K} 4 \mathrm{Fe}(\mathrm{CN}) 6,1 \mathrm{mg} / \mathrm{ml}$ 5-bromo-4-chloro-3-indole-6-D-galactosidase) and incubated over night at $37^{\circ} \mathrm{C}$ in a humidified chamber. After washing in permeabilization solution $3 \times 30$ minutes, sections were counterstained with Nuclear-Fast Red solution (Sigma), dehydrated and mounted in Pertex (Histolab).

\section{Tumor grade assessment}

Tumor tissue from MMTV-PyMT;Pdgfc ${ }^{+/+}$, MMTV-PyMT;Pdgfc ${ }^{+/-}$and MMTVPyMT; $P d g f c^{-/-}$mice ( $\mathrm{n}=5$ mice/genotype, 10 tissue sections per mouse) was classified into different degrees of progression by quantifying the area of transformed glands occupied by each stage. Progression follows from normal fat tissue to a "precancerous stage" characterized by premalignant hyperplasia and adenoma (with the retention of some normal ductal and acinar mammary gland morphology), to a more epithelial cell-dense "early carcinoma" with stromal invasion, and finally to an invasive, very dense, high-mitotic index "late-stage carcinoma". Tumors were evaluated for the proportion of mammary fat tissue, hyperplastic tissue, adenoma, early carcinoma and late carcinoma. PDGF-C specific necrosis was described by a pathologist and scored blindly in the samples.

\section{Quantification of metastases}

The lungs of MMTV-PyMT were embedded in paraffin upon tissue fixation. The metastatic burden was assessed by serial sectioning of the entire lung. Following hematoxylin and eosin staining of every 25 th section, the number of metastatic foci ( $>8$ cells in diameter) was determined in at least 15 tissue sections per lung.

\section{Quantitative reverse-transcription PCR}

For RNA isolation and preparation, primary tumors were snap-frozen and stored at $-80{ }^{\circ} \mathrm{C}$; cell cultures were scraped in PBS with a rubber policeman and centrifuged into a pellet. RNA was isolated using RNAeasy MiniKit (Qiagen). cDNA was prepared using iScript cDNA Synthesis Kit (Bio Rad). KAPA SYBR FAST qPCR Kit Master Mix (KAPA Biosystems) was used for quantitative real-time PCR. The mRNA expression was 
normalized to the housekeeping gene L19. For Foxa1, Esr1, Pdgfra, Fsp1, and Acta2, QuantiTect Primer assays (Qiagen) were used.

L19 primer pair (5' to 3'): GGTGACCTGGATGAGAAGGA and TTCAGCTTGTGGATGTGCTC.

Gata3 primer pair (5' to 3'): CAATGCCTGCGGACTCTACC and GGTGGTGGTCTCGACAGTTCG.

\section{Quantitative reverse-transcription PCR array}

RNA isolation and cDNA synthesis was prepared as described above. qPCR array analyzing 84 mammary cancer specific genes was run according to the manufacturer's instruction (Mouse Breast cancer RT ${ }^{2}$ Profiler ${ }^{\mathrm{TM}}$ PCR array, Qiagen). In brief, cDNA was mixed with 2xRT ${ }^{2}$ SYBR Green ROX FAST Mastermix and diluted with RNase-free $\mathrm{H}_{2} \mathrm{O}$ and added to the $\mathrm{RT}^{2}$ Profiler PCR Array. The PCR was run for 40 cycles; $95^{\circ} \mathrm{C}$ for $15 \mathrm{~s}$ and $60^{\circ}$ for $30 \mathrm{~s}$. Five separate tumors from transplanted FVB/N wt mice from MMTV-PyMT; $P d g f c^{+/+}$and MMTV-PyMT; $P d g f c^{-/}$respectively were run in the qPCR array.

\section{Western blot}

A piece of tumor was minced in liquid $\mathrm{N}_{2}$ before homogenization in lysis buffer $(50 \mathrm{mM}$ Tris- $\mathrm{HCl} \mathrm{pH} 7,4,150 \mathrm{mM} \mathrm{NaCl}, 5 \mathrm{mM}$ EDTA, $1 \%$ Triton-X 100, $1 \%$ SDS with the addition of Roche Complete protease inhibitor and PhosSTOP) using a Precellys 24 instrument (Bertin Technologies). After 30 minutes incubation at $4{ }^{\circ} \mathrm{C}$, followed by centrifugation for 10 minutes at $16000 \times \mathrm{g}$, the supernatant was collected and further subjected to sonication using Vibra-cell ${ }^{\mathrm{TM}}$ (Sonics \& Materials, Inc.). The lysate was spun down at $16000 \times \mathrm{g}$ and the pellet discarded. Protein concentration was determined using Pierce BCA Protein Assay Kit and measurement of the absorption at $562 \mathrm{~nm}$ on a spectrophotometer. The protein suspension was mixed with $5 \times$ NuPAGE $^{\circledR}$ LDS Sample Buffer and NuPAGE ${ }^{\circledR}$ Sample Reducing Agent (Life Technologies), denaturated at $96{ }^{\circ} \mathrm{C}$ for 10 minutes and separated by SDS-PAGE on a NuPAGE ${ }^{\circledR} 4-12 \%$ polyacrylamide gel (Life Technologies). The proteins were transferred to a nitrocellulose filter using a dry blotting system (iBlot ${ }^{\circledR} 2$, Life Technologies). The membrane was blocked 1 hour with 5\% BSA in TBST (0.1\% Tween-20 in TBS) and incubated $\mathrm{o}$ at $4^{\circ} \mathrm{C}$ with primary antibody (FoxA1, 1:200, sc-6553 Santa Cruz Biotechnology Inc; ERa, 1:200, sc-542 Santa Cruz Biotechnology Inc; $\beta$-actin 1:5000, ab-8227 Abcam) in 5\% BSA in TBST. After washing, secondary antibody was applied in $5 \%$ BSA in TBST and incubated for 1 hour at RT. The membrane was washed and developed with Amersham ECL ${ }^{\text {TM }}$ Prime Western Blotting Detection Reagent (GE Healthcare Life Sciences). Luminescence signal was measured using FluorChem ${ }^{\circledR} \mathrm{Q}$ imaging system (Alpha Innotech).

\section{Cell culture}

All cells were tested and found to be negative for mycoplasma infection routinely. PeRoBas1 cells and PeRo-Lum1 cells were derived from tumors in 12 weeks old MMTV-PyMT; Pdgfc ${ }^{+/+}$mouse and MMTV-PyMT; Pdgfc $c^{-/}$mouse, respectively. Human MDA-MB-231 and MCF7 cells were obtained freshly from the American Tissue Type Collection. CAF2 is 
an immortalized cell line of human fibroblasts conditioned by tumor cells in vivo in mice67. All cells were maintained in culture in DMEM Glutamax (Invitrogen), supplemented with $1 \%$ Penicillin/Streptomycin and $10 \%$ fetal bovine serum.

\section{Gene expression analyses of human breast tumors and cell lines}

RNA-seq data from 1086 breast tumors included in The Cancer Genome Atlas project were analyzed for gene expression and correlation to molecular subtype (http:// cancergenome.nih.gov/, downloaded January $30^{\text {th }}$ 2015). Using R (3.1.1), the data were $\log 2$ transformed and breast cancer subtypes were classified using the PAM50 centroids 68 after centering the data around the median. For analysis of gene expression in breast cancer cell lines, data for 51 breast cancer cell lines was obtained from 69. Probe-level data were merged on Entrez Gene IDs, centered, $\log 2$ transformed, and plotted using MedCalc version 11 (MedCalc Software, Belgium). For transcriptional analysis of genes induced by the stimulation of PDGF-CC in CAFs, starved CAF2 cells were induced for $48 \mathrm{~h}$ with $100 \mathrm{ng} / \mathrm{ml}$ of PDGF-CC. Next, RNA was extracted as detailed above and samples with RIN $>9$ were subjected to cDNA library preparation and RNA-seq. The data is available for download from the NCBI Geo database with accession\# GSE108835.

\section{In vitro cell culture assays}

Candidate proteins for mediating the effect of PDGF-CC on the molecular subtype of breast cancer cells were selected from a list of genes predicted to encode secreted proteins (Gene Ontology terms: Extracellular space and Extracellular region) and found to be upregulated in CAF2 cells following stimulation with PDGF-CC (see above).

$3 \times 10^{6} \mathrm{PR}$-Lum1 cells were seeded in culture medium. After 24 hours, the cells were starved for 24 hours in DMEM Glutamax (Invitrogen), supplemented with 1\% bovine serum albumin (BSA; Sigma Aldrich). The cells were next stimulated with rhSTC1 (400 ng/mL; BioVendor), rmHGF (30 ng/mL;), rmIGFBP3 (250 ng/mL; R\&D), or combinations of these factors in starvation medium for 48 hours. The cell line CAF2 was used to produce CAFconditioned medium by incubation for 48 hours in starvation medium. For 4-OH-tamoxifen treatment, 15000 cells were seeded in 96-well plates. After 24 hours incubation in growth medium, followed by 24 hours starvation, the cells were stimulated either with CAFconditioned medium or recombinant factors, as described before. The cells were treated with increasing concentration of 4-OH-tamoxifen (0-5 $\mu \mathrm{M}$; Sigma Aldrich) in the respective stimulation medium at day 4 and 6 post-seeding. The cell proliferation reagent WST-1 (Roche) was used for the viability assay at day 7.

\section{Genomic analysis of breast cancer cell lines}

PeRo-Bas1 and PeRo-Lum1 cells were seeded in normal DMEM growth media for $8 \mathrm{~h}$ (\#10-013-CVR, Corning supplemented with 10\% FBS and 1XPEST) and subsequently starved for 24h in serum-free DMEM (\#10-014-CVR, Corning, supplemented with 1\% Bovine Serum Albumin (Sigma-Aldrich) and 1XPEST). Cells were treated in starvation media using $5 \mu \mathrm{M}$ estradiol (E2758, Sigma-Aldrich) and/or $5 \mu \mathrm{M}$ 4-OH-tamoxifen (SigmaAldrich) for 48 hours before RNA isolation (RNeasy ${ }^{\circledR}$ Mini Kit, \#74106, Qiagen). EtOH was used as vehicle control and treatment medium was replenished every 24 hours. Samples 
prepared in quintuplicate biological repeats were checked for quality using a 2100

Bioanalyzer instrument (Agilent) (RIN range 9.6-10), used as templates to prepare libraries (TruSeq, Illumina), and subjected to RNA sequencing using a NextSeq instrument (Illumina). Raw sequencing data was de-multiplexed and merged into two fastq files (read 1 and read 2) for each sample using Picard tools ExtractIlluminaBarcodes and lluminaBasecallsToFastq (https://broadinstitute.github.io/picard/). Kallisto (v0.43.0)70 was used for abundance estimation and differential expression analysis was performed with Sleuth (v0.29.0)71. Kallisto was run with parameters --bootstrap-samples 100, --bias, --rfstranded and the transcript target was the Gencode release M15 protein-coding transcript sequences. The resulting lists of the top 1000 differentially upregulated and 1000 downregulated genes in $P d g f c$-deficient PeRo-Lum1 cells compared to Pdgfc-proficient PeRo-Bas1 cells were used to investigate the enrichment of regulated genes with ESR1, FOXA1 and GATA3 DNA binding sites, as determined by publicly available datasets from ChIP-seq analysis. ChIP-Seq datasets were obtained through the CistromeDB portal72 and included E2-treated MCF-7 cells73: ESR1, FOXA1, and GATA3 (GSM589237, GSM659787, and GSM720422, respectively) and E2-treated T47D cells73: ESR1 and FOXA1 (GSM589239, and GSM659795, respectively). ChIP-Seq peaks were assigned to GRCh38 Ensembl gene transcripts (gencode_basic=TRUE, biotype=protein coding and miRNAs) and scored using the ClosestGene method74. The ClosestGene method assigns a ChIP-seq peak to the closest transcription start site, and scores the peak based on the empirical cumulative distribution of peaks around the transcription start site. Transcript scores were summed on the gene level and used to examine the enrichment of binding in the top up and down-regulated genes observed in the experiment. From the human ChIP-seq dataset we extracted 15140 genes with homology between human and mouse using BiomaRt (filtering on ortholog_one2one, and homolog_orthology_confidence=1). The ChIP-Seq enrichment test was then confined to the intersection of genes with human/mouse homology and the 13000 genes with detectable expression in the experiment $(\mathrm{n}=11300)$. The summed score and number of genes with ChIP-Seq peaks of the top 1000 differentially expressed genes was compared to $10^{5}$ randomly sampled genelists. The sampled genelists were drawn from the pool of 11300 genes to provide a relevant background. The number of bound genes and sum of scores in each re-sampled gene list was recorded and then ranked (the gene list with the highest score= rank 1) together with the results for the query lists. The rank of the query gene list divided by the total number of re-sample instances was then used as a $\mathrm{p}$ value for the probability to acquire the level of enrichment by chance.

For the analysis of gene expression in cell cultures following estradiol or tamoxifen treatment, we ranked all genes according to the Wald test statistic for differential expression. The Pearson's $r$ correlation test was used to rank the genes that correlate to PDGFC within The Cancer Genome Atlas (TCGA) Breast carcinoma dataset (BRCA). GSEA was performed using the GseaPreranked tool in GSEA v3.075,76 with 1000 random sample permutations, and utilizing the Molecular Signatures Database (MSigDB) hallmark (h.all.v6.1) and curated (c2.all.v6.1) gene sets. The GSEA statistics (Normalized Enrichment Score (NES), nominal P value and FDR q value) are presented in the figures. 


\section{Statistical analyses}

All values depicted represent mean \pm standard error of the mean. Statistical calculations were performed using IBM SPSS Statistics (version 22.0, IBM, Armonk, NY, USA) or GraphPad Prism 7 software. Statistical tests were applied as indicated in the figure legends in a two-sided, unpaired fashion with $\mathrm{p}<0.05$ considered significant. The variance was similar between experimental groups in each experiment, unless otherwise stated. In vivo experiments included cohorts of the size indicated in each figure legend, but at least 6 mice per group. Sample size was chosen by an estimate of the number of animals needed to detect a $20 \%$ difference in the primary endpoint of tumor growth rate, based on the experience of the authors. No animals entering the experimental/intervention phase were excluded from analysis. In vitro analyses were repeated 3-6 times to ensure reproducible conclusions, the exact number of repetitions is stated in each figure legend.

\section{Data availability}

Data from the transcriptional analysis of CAF2 cells stimulated with PDGF-CC is available through the NCBI GEO database with accession number GSE108835.

\section{Supplementary Material}

Refer to Web version on PubMed Central for supplementary material.

\section{Acknowledgement}

K Pietras is the Göran \& Birgitta Grosskopf Professor at Lund University. The research presented herein was supported by grants from the following agencies to K Pietras: a Consolidator Grant from the European Research Council (the TUMORGAN project), the Swedish Research Council, the Swedish Cancer Society, the STARGET consortium (a Swedish Research Council Linnaeus network), BioCARE and Lund University. U Eriksson acknowledges funding support from the Swedish Research Council, the Swedish Cancer Society, Karolinska Institutet and Ludwig Institute for Cancer Research. AMScott acknowledges funding support from NHMRC Fellowship 1084178 and Grant 10927888 and the Operational Infrastructure Support Program provided by the Victorian Government, Australia. We gratefully acknowledge expert help with pathology assessments from the late Dr Dorthe Grabau and provision of the Tam2Y cohort by Dr Mårten Fernö. Further, we would like to thank Diana Cao, Megan O'Brien and Carmel Murone for their technical support and Pär-Ola Bendahl for statistical assistance.

\section{References}

1. Perou CM, et al. Molecular portraits of human breast tumours. Nature. 2000; 406:747-752. [PubMed: 10963602]

2. Goldhirsch A, et al. Thresholds for therapies: highlights of the St Gallen International Expert Consensus on the primary therapy of early breast cancer 2009. Ann Oncol. 2009; 20:1319-1329. [PubMed: 19535820]

3. Sorlie T, et al. Gene expression patterns of breast carcinomas distinguish tumor subclasses with clinical implications. Proc Natl Acad Sci U S A. 2001; 98:10869-10874. [PubMed: 11553815]

4. Goldhirsch A, et al. Personalizing the treatment of women with early breast cancer: highlights of the St Gallen International Expert Consensus on the Primary Therapy of Early Breast Cancer 2013. Ann Oncol. 2013; 24:2206-2223. [PubMed: 23917950]

5. Goldhirsch A, et al. Strategies for subtypes--dealing with the diversity of breast cancer: highlights of the St. Gallen International Expert Consensus on the Primary Therapy of Early Breast Cancer 2011. Ann Oncol. 2011; 22:1736-1747. [PubMed: 21709140]

6. Prat A, et al. Clinical implications of the intrinsic molecular subtypes of breast cancer. Breast. 2015; 24(Suppl 2):S26-35. [PubMed: 26253814] 
7. Ignatiadis M, Sotiriou C. Luminal breast cancer: from biology to treatment. Nat Rev Clin Oncol. 2013; 10:494-506. [PubMed: 23881035]

8. Voduc KD, et al. Breast cancer subtypes and the risk of local and regional relapse. J Clin Oncol. 2010; 28:1684-1691. [PubMed: 20194857]

9. Prat A, Perou CM. Deconstructing the molecular portraits of breast cancer. Mol Oncol. 2011; 5:523. [PubMed: 21147047]

10. Hanahan D, Coussens LM. Accessories to the crime: functions of cells recruited to the tumor microenvironment. Cancer Cell. 2012; 21:309-322. [PubMed: 22439926]

11. Pietras K, Ostman A. Hallmarks of cancer: interactions with the tumor stroma. Exp Cell Res. 2010; 316:1324-1331. [PubMed: 20211171]

12. Kalluri R, Zeisberg M. Fibroblasts in cancer. Nat Rev Cancer. 2006; 6:392-401. [PubMed: 16572188]

13. Cortez E, Roswall P, Pietras K. Functional subsets of mesenchymal cell types in the tumor microenvironment. Semin Cancer Biol. 2014; 25:3-9. [PubMed: 24412106]

14. Augsten M. Cancer-associated fibroblasts as another polarized cell type of the tumor microenvironment. Front Oncol. 2014; 4:62. [PubMed: 24734219]

15. Kim HM, Jung WH, Koo JS. Expression of cancer-associated fibroblast related proteins in metastatic breast cancer: an immunohistochemical analysis. J Transl Med. 2015; 13:222. [PubMed: 26163388]

16. Anderberg C, et al. Paracrine signaling by platelet-derived growth factor-CC promotes tumor growth by recruitment of cancer-associated fibroblasts. Cancer Res. 2009; 69:369-378. [PubMed: 19118022]

17. Pietras K, Pahler J, Bergers G, Hanahan D. Functions of paracrine PDGF signaling in the proangiogenic tumor stroma revealed by pharmacological targeting. PLoS Med. 2008; 5:e19. [PubMed: 18232728]

18. Li X, et al. PDGF-C is a new protease-activated ligand for the PDGF a-receptor. Nature Cell Biol. 2000; 2:302-309. [PubMed: 10806482]

19. Cao R, et al. Angiogenesis stimulated by PDGF-CC, a novel member in the PDGF family, involves activation of PDGFR-aa and -ab receptors. FASEB J. 2002; 16:1575-1583. [PubMed: 12374780]

20. Theurillat JP, et al. NY-ESO-1 protein expression in primary breast carcinoma and metastases: correlation with CD8+ T-cell and CD79a+ plasmacytic/B-cell infiltration. Int J Cancer. 2007; 120:2411-2417. [PubMed: 17294444]

21. Falck AK, et al. Biomarker expression and St Gallen molecular subtype classification in primary tumours, synchronous lymph node metastases and asynchronous relapses in primary breast cancer patients with 10 years' follow-up. Breast Cancer Res Treat. 2013; 140:93-104. [PubMed: 23807420]

22. Falck AK, et al. Analysis of and prognostic information from disseminated tumour cells in bone marrow in primary breast cancer: a prospective observational study. BMC Cancer. 2012; 12:403. [PubMed: 22963449]

23. Guy CT, Cardiff RD, Muller WJ. Induction of mammary tumors by expression of polyomavirus middle T oncogene: a transgenic mouse model for metastatic disease. Mol Cell Biol. 1992; 12:954-961. [PubMed: 1312220]

24. Lin EY, et al. Progression to malignancy in the polyoma middle T oncoprotein mouse breast cancer model provides a reliable model for human diseases. Am J Pathol. 2003; 163:2113-2126. [PubMed: 14578209]

25. Ding H, et al. A specific requirement for PDGF-C in palate formation and PDGFR-alpha signaling. Nat Genet. 2004; 36:1111-1116. [PubMed: 15361870]

26. Cancer Genome Atlas: N. Comprehensive molecular portraits of human breast tumours. Nature. 2012; 490:61-70. [PubMed: 23000897]

27. Badve S, et al. FOXA1 expression in breast cancer--correlation with luminal subtype A and survival. Clin Cancer Res. 2007; 13:4415-4421. [PubMed: 17671124]

28. Thorat MA, et al. Forkhead box A1 expression in breast cancer is associated with luminal subtype and good prognosis. J Clin Pathol. 2008; 61:327-332. [PubMed: 18037662] 
29. Neve RM, et al. A collection of breast cancer cell lines for the study of functionally distinct cancer subtypes. Cancer Cell. 2006; 10:515-527. [PubMed: 17157791]

30. Kong SL, Li G, Loh SL, Sung WK, Liu ET. Cellular reprogramming by the conjoint action of ERalpha, FOXA1, and GATA3 to a ligand-inducible growth state. Mol Syst Biol. 2011; 7:526. [PubMed: 21878914]

31. Kojima Y, et al. Autocrine TGF-beta and stromal cell-derived factor-1 (SDF-1) signaling drives the evolution of tumor-promoting mammary stromal myofibroblasts. Proc Natl Acad Sci U S A. 2010; 107:20009-20014. [PubMed: 21041659]

32. Ray PS, et al. FOXC1 is a potential prognostic biomarker with functional significance in basal-like breast cancer. Cancer Res. 2010; 70:3870-3876. [PubMed: 20406990]

33. Chebil G, Bendahl PO, Idvall I, Ferno M. Comparison of immunohistochemical and biochemical assay of steroid receptors in primary breast cancer--clinical associations and reasons for discrepancies. Acta Oncol. 2003; 42:719-725. [PubMed: 14690157]

34. Hammond ME, et al. American Society of Clinical Oncology/College Of American Pathologists guideline recommendations for immunohistochemical testing of estrogen and progesterone receptors in breast cancer. J Clin Oncol. 2010; 28:2784-2795. [PubMed: 20404251]

35. Polyak K. Breast cancer: origins and evolution. J Clin Invest. 2007; 117:3155-3163. [PubMed: 17975657]

36. Gupta PB, et al. Stochastic state transitions give rise to phenotypic equilibrium in populations of cancer cells. Cell. 2011; 146:633-644. [PubMed: 21854987]

37. Lim E, et al. Aberrant luminal progenitors as the candidate target population for basal tumor development in BRCA1 mutation carriers. Nat Med. 2009; 15:907-913. [PubMed: 19648928]

38. Molyneux G, Smalley MJ. The cell of origin of BRCA1 mutation-associated breast cancer: a cautionary tale of gene expression profiling. J Mammary Gland Biol Neoplasia. 2011; 16:51-55. [PubMed: 21336547]

39. Liu S, et al. BRCA1 regulates human mammary stem/progenitor cell fate. Proc Natl Acad Sci U S A. 2008; 105:1680-1685. [PubMed: 18230721]

40. Molyneux G, et al. BRCA1 basal-like breast cancers originate from luminal epithelial progenitors and not from basal stem cells. Cell Stem Cell. 2010; 7:403-417. [PubMed: 20804975]

41. Su Y, et al. Somatic Cell Fusions Reveal Extensive Heterogeneity in Basal-like Breast Cancer. Cell Rep. 2015; 11:1549-1563. [PubMed: 26051943]

42. Yamamoto S, et al. JARID1B is a luminal lineage-driving oncogene in breast cancer. Cancer Cell. 2014; 25:762-777. [PubMed: 24937458]

43. Bernardo GM, et al. FOXA1 represses the molecular phenotype of basal breast cancer cells. Oncogene. 2013; 32:554-563. [PubMed: 22391567]

44. Sflomos G, et al. A Preclinical Model for ERalpha-Positive Breast Cancer Points to the Epithelial Microenvironment as Determinant of Luminal Phenotype and Hormone Response. Cancer Cell. 2016; 29:407-422. [PubMed: 26947176]

45. Tam WL, et al. Protein kinase $\mathrm{C}$ alpha is a central signaling node and therapeutic target for breast cancer stem cells. Cancer Cell. 2013; 24:347-364. [PubMed: 24029232]

46. Meng F, et al. PDGFRalpha and beta play critical roles in mediating Foxq1-driven breast cancer stemness and chemoresistance. Cancer Res. 2015; 75:584-593. [PubMed: 25502837]

47. Jansson S, et al. The three receptor tyrosine kinases c-KIT, VEGFR2 and PDGFRalpha, closely spaced at 4q12, show increased protein expression in triple-negative breast cancer. PLoS One. 2014; 9:e102176. [PubMed: 25025175]

48. Horikawa $S$, et al. PDGFRalpha plays a crucial role in connective tissue remodeling. Sci Rep. 2015; 517948.

49. Kim YJ, et al. MET is a potential target for use in combination therapy with EGFR inhibition in triple-negative/basal-like breast cancer. Int J Cancer. 2014; 134:2424-2436. [PubMed: 24615768]

50. Ho-Yen CM, et al. C-Met in invasive breast cancer: is there a relationship with the basal-like subtype? Cancer. 2014; 120:163-171. [PubMed: 24150964] 
51. Ponzo MG, et al. Met induces mammary tumors with diverse histologies and is associated with poor outcome and human basal breast cancer. Proc Natl Acad Sci U S A. 2009; 106:12903-12908. [PubMed: 19617568]

52. Graveel CR, et al. Met induces diverse mammary carcinomas in mice and is associated with human basal breast cancer. Proc Natl Acad Sci U S A. 2009; 106:12909-12914. [PubMed: 19567831]

53. Gastaldi S, et al. Met signaling regulates growth, repopulating potential and basal cell-fate commitment of mammary luminal progenitors: implications for basal-like breast cancer. Oncogene. 2013; 32:1428-1440. [PubMed: 22562252]

54. Marzec KA, Baxter RC, Martin JL. Targeting Insulin-Like Growth Factor Binding Protein-3 Signaling in Triple-Negative Breast Cancer. Biomed Res Int. 2015; 2015638526.

55. Bissell MJ, Hines WC. Why don't we get more cancer? A proposed role of the microenvironment in restraining cancer progression. Nat Med. 2011; 17:320-329. [PubMed: 21383745]

56. Marsh T, Pietras K, McAllister SS. Fibroblasts as architects of cancer pathogenesis. Biochim Biophys Acta. 2013; 1832:1070-1078. [PubMed: 23123598]

57. Gascard P, Tlsty TD. Carcinoma-associated fibroblasts: orchestrating the composition of malignancy. Genes Dev. 2016; 30:1002-1019. [PubMed: 27151975]

58. Brechbuhl HM, et al. Fibroblast Subtypes Regulate Responsiveness of Luminal Breast Cancer to Estrogen. Clin Cancer Res. 2017; 23:1710-1721. [PubMed: 27702820]

59. Ozdemir BC, et al. Depletion of carcinoma-associated fibroblasts and fibrosis induces immunosuppression and accelerates pancreas cancer with reduced survival. Cancer Cell. 2014; 25:719-734. [PubMed: 24856586]

60. Rhim AD, et al. Stromal elements act to restrain, rather than support, pancreatic ductal adenocarcinoma. Cancer Cell. 2014; 25:735-747. [PubMed: 24856585]

61. Theurillat JP, et al. NY-ESO-1 protein expression in primary breast carcinoma and metastases: correlation with CD8+ T-cell and CD79a+ plasmacytic/B-cell infiltration. Int J Cancer. 2007; 120:2411-2417. [PubMed: 17294444]

62. Falck AK, et al. Biomarker expression and St Gallen molecular subtype classification in primary tumours, synchronous lymph node metastases and asynchronous relapses in primary breast cancer patients with 10 years' follow-up. Breast Cancer Res Treat. 2013; 140:93-104. [PubMed: 23807420]

63. Falck AK, et al. Analysis of and prognostic information from disseminated tumour cells in bone marrow in primary breast cancer: a prospective observational study. BMC Cancer. 2012; 12:403. [PubMed: 22963449]

64. Chebil G, Bendahl PO, Idvall I, Ferno M. Comparison of immunohistochemical and biochemical assay of steroid receptors in primary breast cancer--clinical associations and reasons for discrepancies. Acta Oncol. 2003; 42:719-725. [PubMed: 14690157]

65. Guy CT, Cardiff RD, Muller WJ. Induction of mammary tumors by expression of polyomavirus middle T oncogene: a transgenic mouse model for metastatic disease. Mol Cell Biol. 1992; 12:954-961. [PubMed: 1312220]

66. Ding H, et al. A specific requirement for PDGF-C in palate formation and PDGFR-alpha signaling. Nat Genet. 2004; 36:1111-1116. [PubMed: 15361870]

67. Kojima Y, et al. Autocrine TGF-beta and stromal cell-derived factor-1 (SDF-1) signaling drives the evolution of tumor-promoting mammary stromal myofibroblasts. Proc Natl Acad Sci U S A. 2010; 107:20009-20014. [PubMed: 21041659]

68. Parker JS, et al. Supervised risk predictor of breast cancer based on intrinsic subtypes. J Clin Oncol. 2009; 27:1160-1167. [PubMed: 19204204]

69. Neve RM, et al. A collection of breast cancer cell lines for the study of functionally distinct cancer subtypes. Cancer Cell. 2006; 10:515-527. [PubMed: 17157791]

70. Bray NL, Pimentel H, Melsted P, Pachter L. Near-optimal probabilistic RNA-seq quantification. Nat Biotechnol. 2016; 34:525-527. [PubMed: 27043002]

71. Pimentel H, Bray N, Puente S, Melsted P, Pachter L. Differential analysis of RNA-Seq incorporating quantification uncertainty. bioRxiv. 2016; doi: 10.1101/058164

72. Mei S, et al. Cistrome Data Browser: a data portal for ChIP-Seq and chromatin accessibility data in human and mouse. Nucleic Acids Res. 2017; 45:D658-D662. [PubMed: 27789702] 
73. Kong SL, Li G, Loh SL, Sung WK, Liu ET. Cellular reprogramming by the conjoint action of ERalpha, FOXA1, and GATA3 to a ligand-inducible growth state. Mol Syst Biol. 2011; 7:526. [PubMed: 21878914]

74. Sikora-Wohlfeld W, Ackermann M, Christodoulou EG, Singaravelu K, Beyer A. Assessing computational methods for transcription factor target gene identification based on ChIP-seq data. PLoS Comput Biol. 2013; 9:e1003342. [PubMed: 24278002]

75. Subramanian A, et al. Gene set enrichment analysis: a knowledge-based approach for interpreting genome-wide expression profiles. Proc Natl Acad Sci U S A. 2005; 102:15545-15550. [PubMed: 16199517]

76. Mootha VK, et al. PGC-1alpha-responsive genes involved in oxidative phosphorylation are coordinately downregulated in human diabetes. Nat Genet. 2003; 34:267-273. [PubMed: 12808457] 

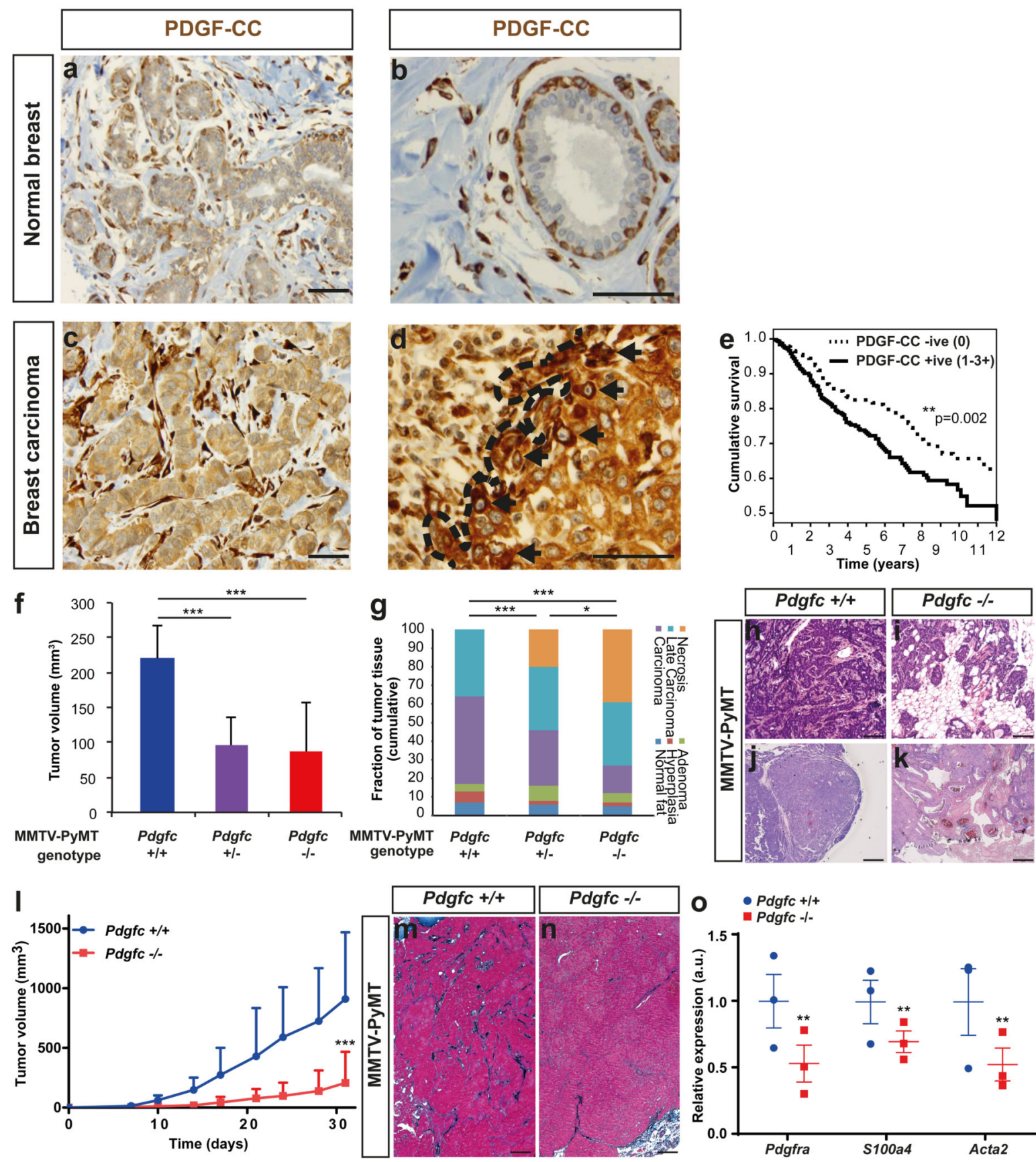

Figure 1. Epithelial expression of PDGF-CC is associated with poor outcome in human breast carcinoma.

a-d, Immunostaining of normal breast tissue (a-b) or breast carcinoma (c-d) for PDGF-CC. Appropriate validation on $>10$ independent samples was performed to ensure reproducible staining pattern on human tissue. Dotted line represents the epithelium-stroma boundary and arrows identify tumor cells with particularly high expression of PDGF-CC on the invasive border. Scale bar, $40 \mu \mathrm{m}$. e, Kaplan-Meier analysis of breast cancer-specific survival dichotomized according to absence (negative, $n=438$ samples) or presence (positive, defined 
as a score of $1+$ to $3+, n=452$ samples) of PDGF-CC in tumor cells (for scoring scheme, see Supplementary Fig. 1a) of breast carcinomas from the Zürich cohort. ** $\mathrm{p}=0.002$, log-rank test $\mathbf{f}$, Average volume (with SEM) of tumors from 14 weeks old MMTV-PyMT mice ( $\mathrm{n}=10$ animals in each group). $* * * \mathrm{p}=0.0001$, two-sided, unpaired, equal variance Student's t-test. g, Grading of tumors from 14 weeks old MMTV-PyMT mice ( $\mathrm{n}=5$ animals in each group). * Distribution of $P d g f c^{-/-}$vs $P d g f c^{+/-}$p $=0.036, \chi^{2}$ test. *** Distribution of $P d g f c^{-/-}$vs $P d g f c^{+/+}$ $\mathrm{p}=3 * 10^{-11}$ and distribution of $P d g f c^{+/-}$vs $P d g f^{+/+} \mathrm{p}=0.00052, \chi^{2}$ test. h-k, Hematoxylin and eosin staining of tumors from MMTV-PyMT mice at 14 weeks of age. Scale bar, 500 $\mu \mathrm{m}$. l, Average volume (with SEM) of tumors derived from MMTV-PyMT mice transplanted into the mammary fat pad of wt mice ( $n=21$ animals in each group, composed of $n=7$ animals each from 3 independent experiments). $* * * \mathrm{p}=6 * 10^{-6}$, two-sided, unpaired, equal variance Student's t-test. m-n, Masson's tri-chrome staining of tissue sections from tumors from 14 weeks old MMTV-PyMT mice. Representative pictures from the analysis of $n=10$ animals from each group are shown. Scale bar, $500 \mu \mathrm{m}$. o, Quantitative real-time PCR analysis of the average expression (with SEM) of markers for cancer-associated fibroblasts in tumor lysates of tumors from 14 weeks old MMTV-PyMT mice ( $\mathrm{n}=3$ animals in each group, analysis performed independently 3 times). ** Pdgfra, $\mathrm{p}=0.007 ;$ S100a4, $\mathrm{p}=0.003$; Acta2, $\mathrm{p}=0.009$ two-sided, unpaired, equal variance Student's t-test. 

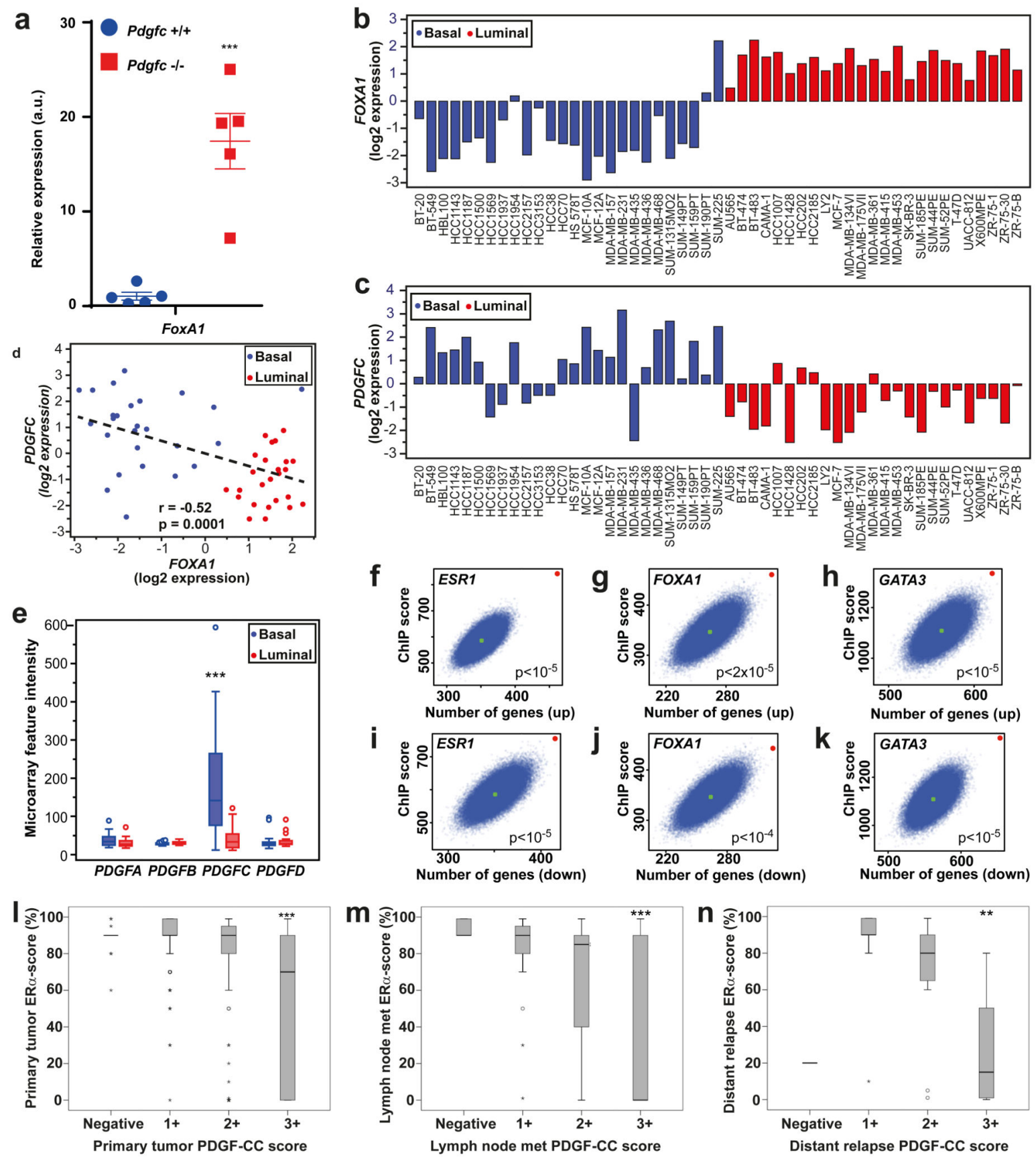

Figure 2. Expression of PDGF-CC in breast carcinomas is associated with the basal-like molecular subtype and hormone receptor negativity.

a, Quantitative RT-PCR analysis of the luminal subtype marker Foxa1 in tumors from 14 weeks old MMTV-PyMT mice ( $\mathrm{n}=5$ animals in each group, analysis performed independently 3 times). $* * * \mathrm{p}<0.001$, two-sided, unpaired, equal variance Student's t-test. bc, Expression of FOXA1 (b) and PDGFC (c) in a panel of 51 breast cancer cell lines 29 divided according to basal-like or luminal-like molecular subtype. d, Correlation between expression of FOXA1 and $P D G F C$ in a panel of 51 breast cancer cell lines divided according 
to basal-like or luminal-like molecular subtype. Correlation coefficient and p-value from Pearson correlation analysis of $n=26$ basal cell lines and $n=25$ luminal cell lines. $\mathbf{e}$, Expression of all PDGF family members in $n=26$ basal cell lines and $n=25$ luminal cell lines. Box represents the interquartile range, line represents the average expression and whiskers depict range of expression with statistical outliers indicated ( $>2$ standard deviations from the mean). $* * * \mathrm{p}=1 * 10^{-6}$, two-sided, unpaired Welch's unequal variances t-test. f-k, Enrichment of luminal-defining transcription factors ESR1, FOXA1, and GATA3 binding sites, as derived from public datasets of ChIP-seq analysis in MCF7 cells, in the 1000 most up/down-regulated genes in $P d g f c$-deficient PeRo-Lum1 cells compared to $P d g f c$-proficient PeRo-Bas 1 cells. Red, query signature; blue, $\mathrm{n}=10^{5}$ randomly sampled gene lists of equal size to the query signature; green, average of the randomly sampled gene lists. The rank of the query gene list divided by the total number of re-sample instances was then used as a pvalue for the probability to acquire the level of enrichment by chance. l-n, Correlation between expression of ERa and PDGF-CC in primary breast carcinomas (1, $\mathrm{n}=470$ independent samples, ${ }^{* * *} \mathrm{p}=2.1 * 10^{-14}$ independent samples, two-sided Jonckheere-Terpstra test for ordered alternatives), synchronous lymph nodes ( $\mathrm{m}, \mathrm{n}=132$ independent samples, $* * * \mathrm{p}=1.5^{*} 10^{-8}$ independent samples, two-sided Jonckheere-Terpstra test for ordered alternatives) or asynchronous distant replapses ( $\mathrm{n}, \mathrm{n}=29$ independent samples, ${ }^{* *} \mathrm{p}=0.0015$ independent samples, two-sided Jonckheere-Terpstra test for ordered alternatives) included in the Lund cohort. Box represents the interquartile range, line represents the median expression and whiskers depict 1.5x the height of the box. Circles and stars represent statistical outliers that fall outside of the whiskers and extreme outliers that fall outside of $3 x$ the height of the box, respectively. 

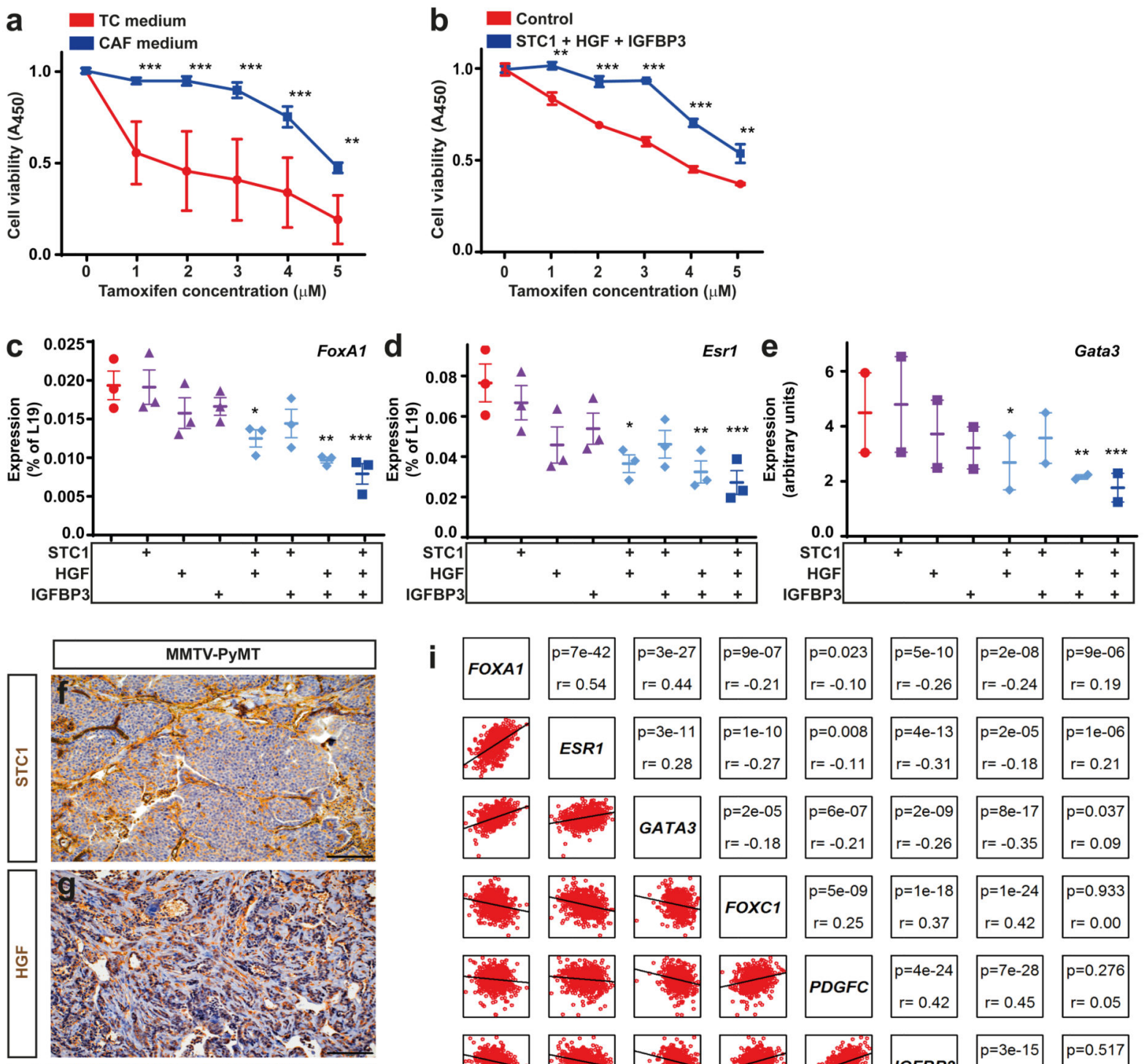

i
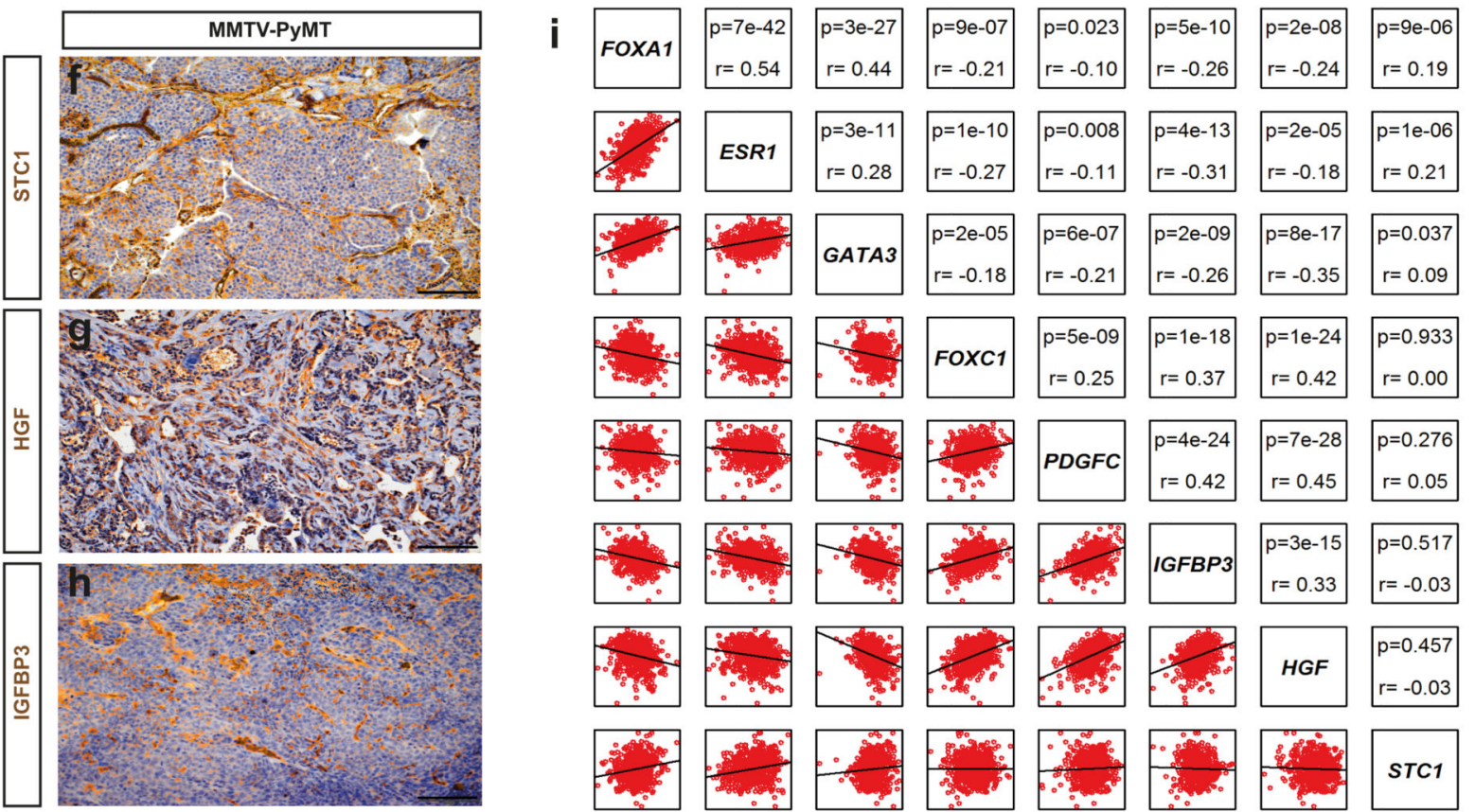

Figure 3. CAF-derived factors induced by PDGF-CC reduce the sensitivity of breast tumor cells to endocrine therapy.

a, Viability of PeRo-Lum1 luminal breast cancer cells derived from MMTV-PyMT; Pdgfc ${ }^{-/-}$ mice in the presence of increasing concentrations of 4-OH-tamoxifen in medium conditioned by tumor cells (TC) or CAFs (the average (with SEM) of $n=6$ independent experiments is shown). ** $\mathrm{p}<0.01$, *** $\mathrm{p}<0.001$, ( $\mathrm{p}$-values for control vs STC1+HGF + IGFBP3 for each tamoxifen concentration (1-5 $\mu \mathrm{M}=0.0001,0.00001,0.00001,0.00001$, 0.005 , respectively), two-way ANOVA with p-values from Bonferroni post-hoc test. $\mathbf{b}$, 
Viability of PeRo-Lum1 cells in the presence of increasing concentrations of 4-OHtamoxifen in medium conditioned by nothing or STC1, HGF and IGFBP3 (the average (with SEM) of $\mathrm{n}=6$ independent experiments is shown). $* * \mathrm{p}<0.01, * * * \mathrm{p}<0.001$ ( $\mathrm{p}$-values for control vs STC1+HGF+IGFBP3 for each tamoxifen concentration $(1-5 \mu \mathrm{M}=0.003,0.0003$, $0.00008,0.0002,0.005$, respectively), two-way ANOVA with p-values from Bonferroni post-hoc test. c-e, Quantitative RT-PCR analysis of the average expression (with SEM) of Foxa1 (c, n=3 independent experiments), Esr 1 (d, n=3 independent experiments) or Gata3 (e, $n=2$ independent experiments) in PeRo-Lum1 cells in the presence of combinations of STC1, HGF and IGFBP3. Foxa $1 * \mathrm{p}=0.03, * * \mathrm{p}=0.002, * * * \mathrm{p}=0.0003$, one-way ANOVA with p-values from Bonferroni post-hoc test. $E s r 1 * \mathrm{p}=0.01, * * \mathrm{p}=0.005, * * * \mathrm{p}=0.001$, oneway ANOVA with $\mathrm{p}$-values from Bonferroni post-hoc test. Gata3* $\mathrm{p}=0.02, * * \mathrm{p}=0.007$, *** $\mathrm{p}=0.001$, one-way ANOVA with $\mathrm{p}$-values from Bonferroni post-hoc test. $\mathbf{f}-\mathbf{h}$, Immunostaining of tissue sections from tumors from 14 weeks old MMTV-PyMT mice for STC1 (f), HGF (g) or IGFBP3 (h). Immunostaining was performed $n=3$ independent times and representative images from $\mathrm{n}=5$ tumors are shown. Scale bar, $100 \mu \mathrm{m}$. i, Pearson's correlation analysis of factors denoting luminal-like (FOXA1, ESR1, GATA3) or basal-like (FOXC1, PDGFC) molecular subtype in luminal subtype breast carcinomas included in the TCGA cohort ( $\mathrm{n}=1086$ independent tumors included in analysis). 

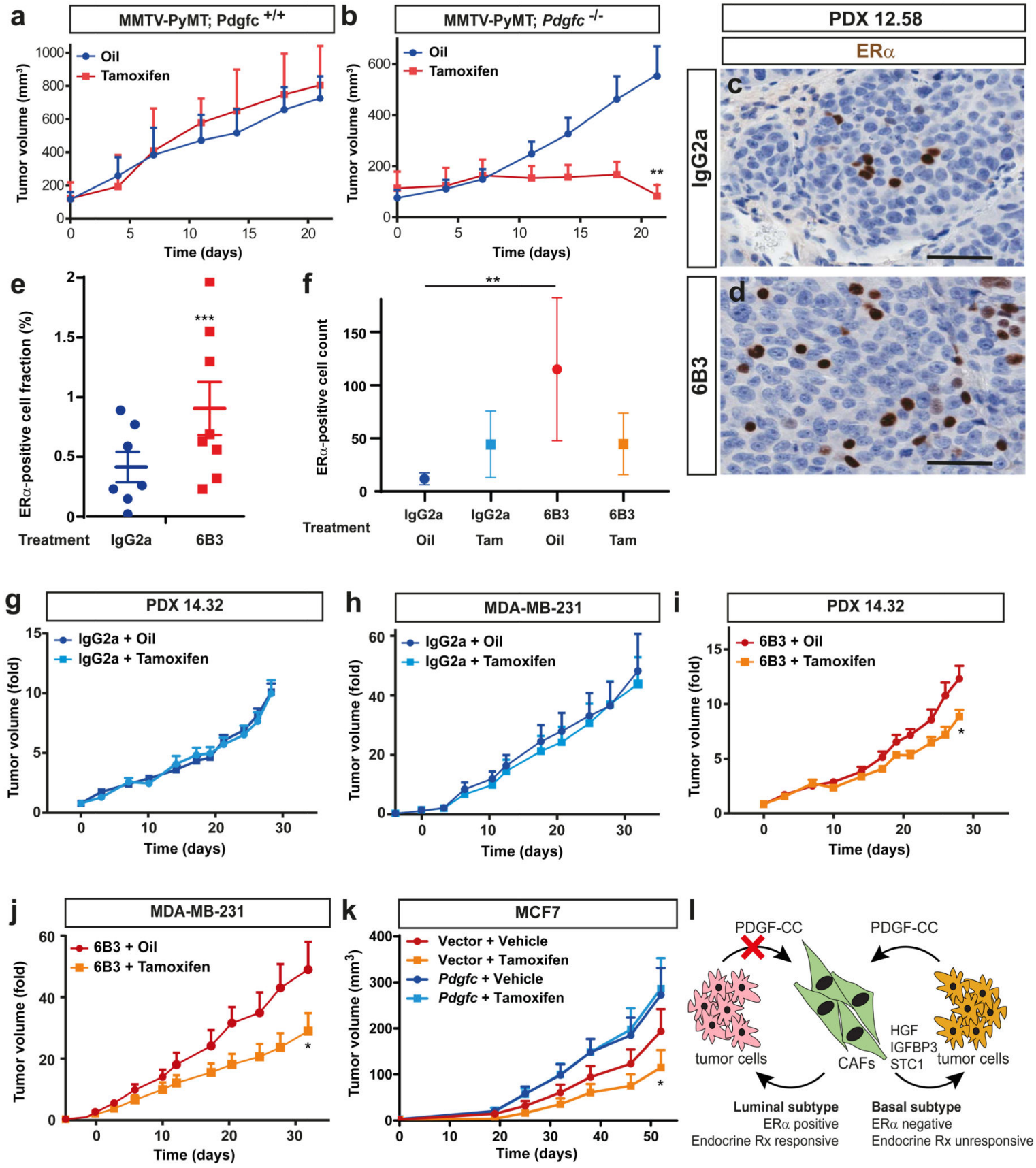

Figure 4. Genetic or pharmacological targeting of PDGF-CC induces expression of ERa and sensitizes tumors to endocrine therapy.

$\mathbf{a}-\mathbf{b}$, Treatment of wt mice transplanted orthotopically with tumors derived from 14 weeks old MMTV-PyMT; $P d g f c^{+/+}$mice (a) or MMTV-PyMT; $P d g f c^{-/-}$mice (b) with oil vehicle $\left(P d g f c^{+/+} \mathrm{n}=7\right.$ animals, $P d g f c^{-/-} \mathrm{n}=6$ animals) or tamoxifen $\left(P d g f c^{-+/+} \mathrm{n}=6\right.$ animals, $P d g f c^{-/-}$ $\mathrm{n}=7$ animals). Average tumor volume (with SEM) depicted. ** $\mathrm{p}=0.002$, two-sided, unpaired, equal variance Student's t-test. X-axis shows time after start of therapy. c-d, Immunostaining for ERa (brown) of tissue sections from the triple-negative patient-derived 
xenograft 12.58 from mice treated with control $\operatorname{IgG}$ or $6 \mathrm{~B} 3$. Immunostaining performed $\mathrm{n}=3$ independent times and representative images are shown. Scale bar, $40 \mu \mathrm{m}$. e, Quantification of the average ERa expression (with SEM) in patient-derived xenograft 12.58 from mice treated with control IgG or $6 \mathrm{~B} 3$ (IgG, $\mathrm{n}=7$ animals; $6 \mathrm{~B} 3, \mathrm{n}=8$ animals). *** $\mathrm{p}=7 * 10^{-7}, \chi^{2}$ test. $\mathbf{f}$, Quantification of the average number of ERa-expressing cells (with SEM) in MDAMB-231 tumors from mice treated with control $\mathrm{IgG}$ or $6 \mathrm{~B} 3$ in combination with oil vehicle (IgG, $\mathrm{n}=10$ animals; 6B3, $\mathrm{n}=12$ animals) or tamoxifen (IgG, $\mathrm{n}=12$ animals; $6 \mathrm{~B} 3, \mathrm{n}=12$ animals). ${ }^{* *} \mathrm{p}=0.003$, two-sided, unpaired, equal variance Student's t-test. g, Treatment of mice carrying orthotopically transplanted triple-negative patient-derived xenograft 14.32 with control $\mathrm{IgG}$ in combination with vehicle ( $\mathrm{n}=10$ animals) or tamoxifen ( $\mathrm{n}=10$ animals). Average tumor volume (with SEM) depicted. X-axis shows time after start of therapy. $\mathbf{h}$, Treatment of mice carrying orthotopically transplanted MDA-MB-231 tumors with control IgG in combination with oil vehicle ( $\mathrm{n}=10$ animals) or tamoxifen ( $\mathrm{n}=12$ animals). Average tumor volume (with SEM) depicted. X-axis shows time after start of therapy. i, Treatment of mice carrying orthotopically transplanted triple-negative patient-derived xenograft 14.32 with the neutralizing PDGF-CC antibody 6B3 in combination with vehicle ( $\mathrm{n}=10$ animals) or tamoxifen ( $\mathrm{n}=10$ animals). Average tumor volume (with SEM) depicted. * $\mathrm{p}=0.03$, twosided, unpaired, equal variance Student's t-test. X-axis shows time after start of therapy. $\mathbf{j}$, Treatment of mice carrying orthotopically transplanted MDA-MB-231 tumors with the neutralizing PDGF-CC antibody 6B3 in combination with oil vehicle ( $\mathrm{n}=12$ animals) or tamoxifen ( $\mathrm{n}=13$ animals). Average tumor volume (with SEM) depicted. * $\mathrm{p}=0.025$, twosided, unpaired, equal variance Student's t-test. X-axis shows time after start of therapy. $\mathbf{k}$, Treatment of mice carrying orthotopically transplanted MCF7 tumors infected with lentivirus carrying empty vector or $P d g f c$ vector with oil vehicle (Vector, $\mathrm{n}=9$ animals; $P d g f c, \mathrm{n}=10$ animals) or tamoxifen (Vector, $\mathrm{n}=10$ animals, $P d g f c, \mathrm{n}=8$ animals). Average tumor volume (with SEM) depicted. * Vector + Tamoxifen $v s$ Pdgfc + Tamoxifen, $\mathrm{p}=0.029$, two-sided, unpaired, equal variance, Student's t-test. $\mathrm{X}$-axis shows time after start of therapy. I, Schematic diagram of the paracrine action of PDGF-CC in the breast tumor microenvironment. An active crosstalk between malignant cells and CAFs mediated through PDGF-CC results in specification of the molecular subtype and regulation of sensitivity to endocrine therapy. 


\section{Table 1}

Cox regression analysis of risk factors for breast cancer death. RR - relative risk, $\mathrm{CI}$ - confidence interval, NA - not analyzed, pT - Primary tumor stage, $\mathrm{pN}$ - Number of lymph nodes involved, G - Nottingham histological grade, CK5/6 - cytokeratin 5/6.

\begin{tabular}{|c|c|c|c|c|}
\hline \multirow[b]{2}{*}{ Parameter } & Univariable analysis & \multicolumn{3}{|c|}{ Zürich cohort } \\
\hline & RR $(95 \%$ CI $)$ & P-value & RR $(95 \%$ CI $)$ & P-value \\
\hline \multicolumn{5}{|l|}{ Age at diagnosis } \\
\hline 60 years $\langle v s\rangle=$ & $1.542(1.170-2.032)$ & 0.002 & $1.437(1.035-1.995)$ & 0.03 \\
\hline \multicolumn{5}{|l|}{ Menopausal status } \\
\hline pre $v s$ post & $1.355(0.965-1.902)$ & 0.079 & NA & NA \\
\hline \multicolumn{5}{|l|}{$p T$} \\
\hline $1-4$ & $1.611(1.421-1.825)$ & $<0.001$ & $1.624(1.378-1.913)$ & $<0.001$ \\
\hline \multicolumn{5}{|l|}{$p N$} \\
\hline $0-3$ & $1.681(1.423-1.986)$ & $<0.001$ & $1.415(1.173-1.708)$ & $<0.001$ \\
\hline \multicolumn{5}{|l|}{$G$} \\
\hline $1-3$ & $1.669(1.341-2.077)$ & $<0.001$ & $1.410(1.067-1.862)$ & 0.016 \\
\hline \multicolumn{5}{|l|}{$E R a$} \\
\hline negative $v s$ positive & $0.494(0.364-0.670)$ & $<0.001$ & $1.211(0.757-1.937)$ & 0.424 \\
\hline \multicolumn{5}{|l|}{$\operatorname{PgR}$} \\
\hline negative $v s$ positive & $0.511(0.388-0.673)$ & $<0.001$ & $0.603(0.411-0.886)$ & 0.01 \\
\hline \multicolumn{5}{|l|}{ HER2 } \\
\hline $0-2+v s 3+$ & $1.941(1.373-2.745)$ & $<0.001$ & $1.577(1.041-2.388)$ & 0.031 \\
\hline \multicolumn{5}{|l|}{$C K 5 / 6$} \\
\hline negative $v s$ positive & $1.496(1.010-2.217)$ & 0.045 & $1.457(0.891-2.382)$ & 0.133 \\
\hline \multicolumn{5}{|l|}{ PDGF-CC Epithelium } \\
\hline 0 vs 1-3+ & $1.520(1.156-1.998)$ & 0.003 & $1.484(1.035-2.127)$ & 0.032 \\
\hline \multicolumn{5}{|l|}{ PDGF-CC Stroma } \\
\hline $1+$ vs $2-3+$ & $0.931(0.708-1.225)$ & 0.931 & NA & NA \\
\hline
\end{tabular}




\section{Table 2}

Spearman's rank correlations of PDGF-CC expression and FoxA1 expression with biomarkers for molecular subtypes. Correlation coefficients in bold are statistically significant. NA - not analyzed.

\begin{tabular}{|rrlllll|}
\hline Zürich cohort & & FoxA1 & ERa & PgR & CK5/6 & EGFR \\
\hline \multirow{2}{*}{ PDGF-CC Epithelium } & Correlation coefficient & $\mathbf{- 0 . 1 8 0}$ & $\mathbf{- 0 . 4 8 5}$ & $\mathbf{- 0 . 3 0 1}$ & $\mathbf{0 . 3 3 1}$ & $\mathbf{0 . 3 0 3}$ \\
& $P$-value & 0.002 & 0.0001 & 0.0001 & 0.0001 & 0.0002 \\
& $N$ & 288 & 856 & 854 & 860 & 854 \\
& FoxA1 & & & & & \\
& Correlation coefficient & 1.000 & $\mathbf{0 . 4 5 4}$ & $\mathbf{0 . 2 9 9}$ & $\mathbf{- 0 . 2 4 6}$ & NA \\
& $P$-value & NA & 0.0001 & 0.0001 & 0.0001 & NA \\
$N$ & 315 & 285 & 309 & 310 & NA \\
\hline
\end{tabular}

